\section{OPEN ACCESS}

Edited by:

Hui Wu,

East China University of Science and Technology, China

Reviewed by:

Gennaro Agrimi,

University of Bari Aldo Moro, Italy

Hiroshi Takagi,

Nara Institute of Science

and Technology (NA/ST), Japan

*Correspondence:

Alicia González

amanjarr@ifc.unam.mx

Specialty section:

This article was submitted to

Microbial Physiology and Metabolism,

a section of the journal

Frontiers in Microbiology

Received: 05 May 2020

Accepted: 14 July 2020

Published: 04 August 2020

Citation:

Aguirre-López B,

Escalera-Fanjul X, Hersch-González J,

Rojas-Ortega E, El-Hafidi M, Lezama M, González J, Bianchi MM, López G, Márquez D, Scazzocchio C,

Riego-Ruiz L and González A (2020)

In Kluyveromyces lactis a Pair

of Paralogous Isozymes Catalyze

the First Committed Step of Leucine

Biosynthesis in Either

the Mitochondria or the Cytosol.

Front. Microbiol. 11:1843.

doi: 10.3389/fmicb.2020.01843

\title{
In Kluyveromyces lactis a Pair of Paralogous Isozymes Catalyze the First Committed Step of Leucine Biosynthesis in Either the Mitochondria or the Cytosol
}

\begin{abstract}
Beatriz Aguirre-López ${ }^{1}$, Ximena Escalera-Fanjul², Jaqueline Hersch-González¹, Eréndira Rojas-Ortega', Mohammed El-Hafidi", Mijail Lezama', James González', Michele Maria Bianchi', Geovani López¹, Dariel Márquez", Claudio Scazzocchio ${ }^{5,6}$, Lina Riego-Ruiz ${ }^{7}$ and Alicia González ${ }^{1 *}$
\end{abstract}

\footnotetext{
' Departamento de Bioquímica y Biología Estructural, Instituto de Fisiología Celular, Universidad Nacional Autónoma de México, Mexico City, Mexico, ${ }^{2}$ Theoretical Biophysics, Humboldt-Universität zu Berlin, Berlin, Germany, ${ }^{3}$ Departamento de Biomedicina Cardiovascular, Instituto Nacional de Cardiología Ignacio Chávez, Mexico City, Mexico, ${ }^{4}$ Dipartimento di Biologia e Biotecnologie Charles Darwin, Sapienza Università di Roma, Rome, Italy, ${ }^{5}$ Department of Microbiology, Imperial College London, London, United Kingdom, ${ }^{6}$ Institut de Biologie Intégrative de la Cellule (I2BC), Gif-sur-Yvette, France, ${ }^{7}$ División de Biología Molecular, Instituto Potosino de Investigación Científica y Tecnológica AC, San Luis Potosí, Mexico
}

Divergence of paralogous pairs, resulting from gene duplication, plays an important role in the evolution of specialized or novel gene functions. Analysis of selected duplicated pairs has elucidated some of the mechanisms underlying the functional diversification of Saccharomyces cerevisiae (S. cerevisiae) paralogous genes. Similar studies of the orthologous pairs extant in pre-whole genome duplication yeast species, such as Kluyveromyces lactis ( $K$. lactis) remain to be addressed. The genome of $K$. lactis, an aerobic yeast, includes gene pairs generated by sporadic duplications. The genome of this organism comprises the KILEU4 and KILEU4BIS paralogous pair, annotated as putative $\alpha$-isopropylmalate synthases ( $\alpha$-IPMSs), considered to be the orthologs of the S. cerevisiae SCLEU4/SCLEU9 paralogous genes. The enzymes encoded by the latter two genes are mitochondrially located, differing in their sensitivity to leucine allosteric inhibition resulting in ScLeu4-ScLeu4 and ScLeu4-ScLeu9 sensitive dimers and ScLeu9-ScLeu9 relatively resistant homodimers. Previous work has shown that, in a Scleu4A mutant, ScLEU9 expression is increased and assembly of ScLeu9ScLeu9 leucine resistant homodimers results in loss of feedback regulation of leucine biosynthesis, leading to leucine accumulation and decreased growth rate. Here we report that: (i) $K$. lactis harbors a sporadic gene duplication, comprising the KILEU4, syntenic with S. cerevisiae ScLEU4 and ScLEU9, and the non-syntenic KILEU4BIS, arising from a pre-WGD event. (ii) That both, KILEU4 and KILEU4BIS encode leucine sensitive $\alpha$-IPMSs isozymes, located in the mitochondria (K/Leu4) and the cytosol (KILeu4BIS), respectively. (iii) That both, KILEU4 or KILEU4BIS complement the Scleu4 $\triangle$ Scleu9 $\Delta$ leucine auxotrophic phenotype and revert the enhanced SCLEU9 transcription observed in a Scleu4A ScLEU9 mutant. The Scleu4A ScLEU9 growth mutant 
phenotype is only fully complemented when transformed with the syntenic KILEU4 mitochondrial isoform. KILEU4 and KILEU4BIS underwent a different diversification pathways than that leading to SCLEU4/SCLEU9. KILEU4 could be considered as the functional ortholog of SCLEU4, since its encoded isozyme can complement both the

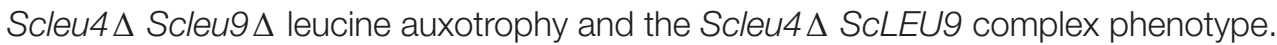

Keywords: gene duplication, functional diversification, oligomerization, feedback control, subcellular localization

\section{INTRODUCTION}

Gene duplication is a source of new or specialized biological functions (Ohno, 1970; Lynch et al., 1991; Force et al., 1999; Kellis et al., 2004). Retained duplicate genes (paralogs) can provide increased dosage of the same product, or may go through a process of sub- or neo-functionalization. In the former, both copies of the gene lose and/or specialize a subset of their ancestral functions, in the latter, at least one of the copies acquires a new function (Ohno, 1970; Lynch et al., 1991; Force et al., 1999).

Paralogous genes can originate from Whole Genome Duplication (WGD) (ohnologs) events or from sporadic duplications known as Small Scale Duplications (SSD) (paralogs). The extant yeast genomes from the Saccharomyces and closely related genera arose from a WGD event within the Saccharomycetaceae family (Kellis et al., 2004). More recent work established that this duplication originated from the fusion of two different species genomes (Marcet-Houben and Gabaldón, 2015). The lineage that gave rise to Kluyveromyces lactis (K. lactis) diverged from the Saccharomyces cerevisiae (S. cerevisiae) lineage before the WGD event (Kellis et al., 2004). Accordingly, this yeast exhibits much less overall genetic redundancy (Conde e Silva et al., 2009), suggesting that the paralogs genes present in K. lactis originated from independent SSD events (Dujon et al., 2004; Huerta-Cepas et al., 2014). Subfunctionalization of paralogs pairs can be achieved through various non-exclusive molecular mechanisms such as modifications of the coding sequence leading to: (i) changes in the kinetic parameters (DeLuna et al., 2001; Rojas-Ortega et al., 2018), (ii) differential subcellular localization (Marques et al., 2008), (iii) formation of hetero-oligomeric isozymes with emerging biochemical properties (DeLuna et al., 2001; López et al., 2015), or (iv) modifications of the regulatory region determining differential expression of each copy (Avendaño et al., 2005; Quezada et al., 2008; González et al., 2017).

The analysis of the functional diversification of orthologous gene pairs from S. cerevisiae and $K$. lactis could allow addressing the question of whether functional diversification of a given paralogous pair follows either the same or distinct diversification pathways in different organisms. An interesting example of duplicated pairs, present in both $S$. cerevisiae and $K$. lactis is that of $\alpha$-isopropylmalate synthases ( $\alpha$-IPMSs). The ScLEU4 (YNL104C) and ScLEU9 (YOR108W) genes from S. cerevisiae form part of a duplicated chromosomal block generated from the WGD event (Wolfe and Shields, 1997), while those present in the K. lactis KlLEU4 (KLLA0F23529G alias KLLA-ORF403) and KlLEU4BIS (KLLLA0D14201G alias KLLA-ORF4593) were originated from an independent gene duplication event prior to the WGD (see below).

The ScLeu4 and ScLeu9 $\alpha$-IPMSs paralogous isozymes from $S$. cerevisiae catalyze the synthesis of $\alpha$-isopropylmalate ( $\alpha$-IPM) from acetyl coenzyme A (acetyl-CoA) and $\alpha$-ketoisovalerate ( $\alpha$ KIV), the first committed step of leucine biosynthesis (Ryan et al., 1973; Roeder and Kohlhaw, 1980; Chang et al., 1984, 1985; Beltzer et al., 1988; Cavalieri et al., 1999; Casalone et al., 2000; Kohlhaw, 2003; Figure 1). The catalytic activities of ScLeu4-ScLeu4 and ScLeu9-ScLeu9 homodimers are feed-back inhibited by leucine, albeit with a different kinetics (see below) (Roeder and Kohlhaw, 1980).

$K$. lactis harbors two ORFS with high similarity to ScLEU4/ScLEU9, KlLEU4, and KlLEU4BIS, whose physiological role has not been previously analyzed, the successive steps of the leucine biosynthetic pathway being identical to that extant in S. cerevisiae. In the latter, the consecutive reactions carried out in the cytoplasm, catalyzed by $S c$ Leu1 and ScLeu2 convert $\alpha$-IPM to $\alpha$-ketoisocaproate $(\alpha$-KIC), which is further converted to leucine by the $S c$ Bat1 and $S c$ Bat2 paralogous aminotransferases (Colón et al., 2011). KLLA0A04961g and KLLA0D04906g are the K. lactis orthologous of ScLEU1 and ScLEU2, respectively. Null Klbat1 $\Delta$ mutations result in valine/isoleucine/leucine auxotrophy (Colón et al., 2011). Additionally, the mitochondrial inner membrane $\alpha$-IPM transporter OacAC1 has also an orthologous counterpart in K. lactis (KLLA0B12826g) (Byrne and Wolfe, 2005), however, its functional role has not been studied.

In S. cerevisiae, the leucine biosynthesis intermediate $\alpha$-IPM plays a dual physiological role. On the one hand, it is the leucine precursor (Chang et al., 1985; Kohlhaw, 2003), on the other, it acts as the co-activator of the ScLeu3 master transcriptional regulator (Kohlhaw, 2003), which modulates the expression of a number of genes within and beyond leucine metabolism (Kohlhaw, 2003). ScLeu3 has a dual function (Kohlhaw, 2003). At high $\alpha$-IPM concentrations it acts as a transcriptional activator of genes involved in branched chain amino acids (Kohlhaw, 2003) and glutamic acid (Hu et al., 1995) biosynthesis. Low intracellular concentrations of $\alpha$-IPM shift its function to that of a transcriptional repressor of other genes (Kohlhaw, 2003). ORF KLLA0D10593 probably encodes a ScLeu3 K. lactis orthologous protein (KlLeu3).

We have previously established how ScLEU4 and ScLEU9 have differentially subfunctionalized (López et al., 2015). In Scleu4 $\Delta$ or Scleu9 $\triangle$ single knockout mutants, homodimeric ScLeu4 or ScLeu9 isozymes are present; however, when ScLEU4 and ScLEU9 are simultaneously expressed, ScLeu4-ScLeu9 heterodimeric enzymes are preferentially assembled. The in vivo assembly 


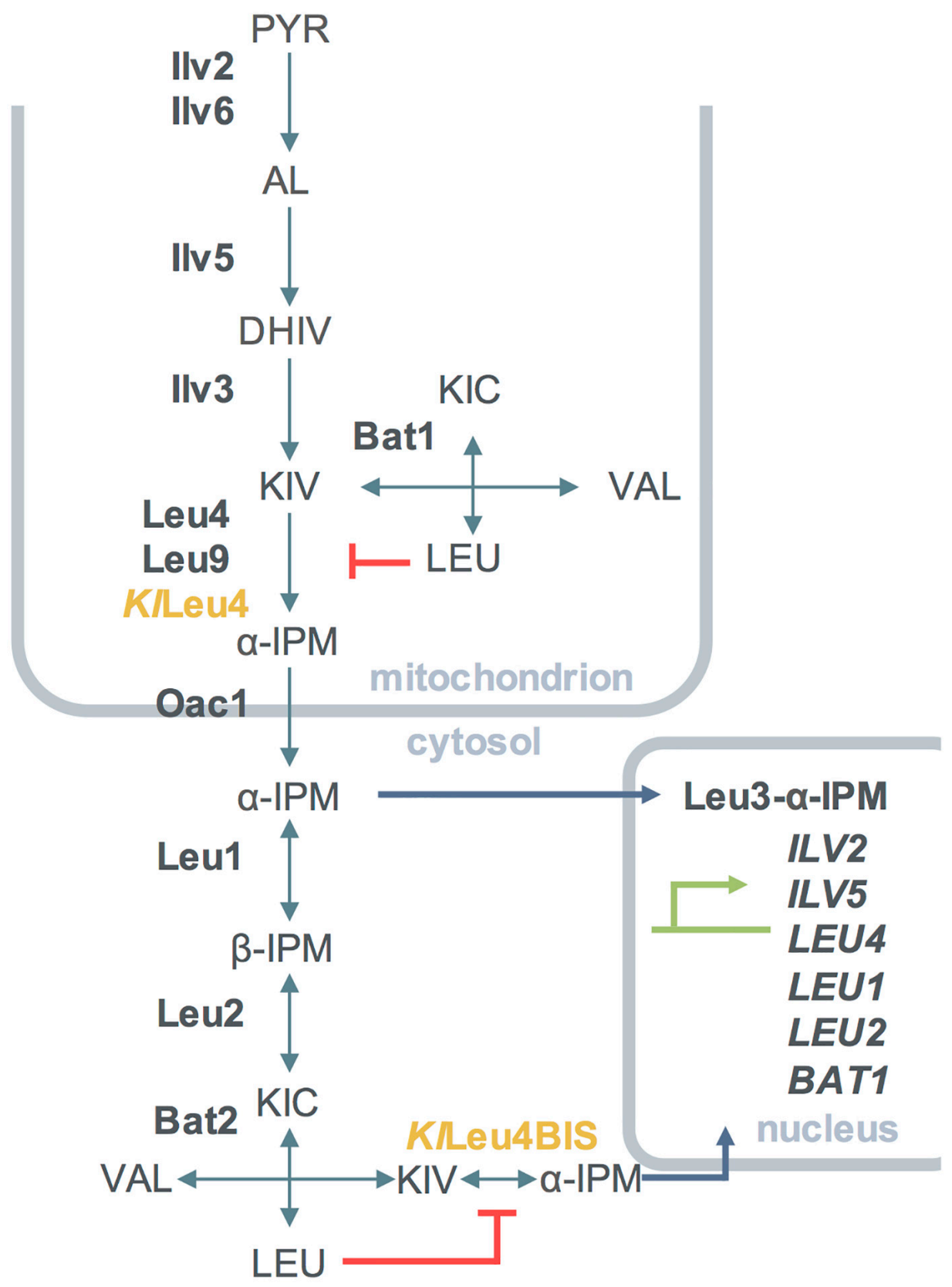

FIGURE 1 | Diagrammatic representation of the compartmentalization of the leucine amino acid biosynthetic pathway of S. cerevisiae including $K$. lactis $\alpha$-IPMSs when transformed in S. cerevisiae. Simple and double arrows indicate reactions considered to be irreversible and reversible, respectively. Enzymes from $K$. lactis are shown in yellow, enzymes form S. cerevisiae are shown in bold font, metabolites are shown in capital letters, genes activated by Leu3- $\alpha-$ IPM are shown in capital italic letters. Enzymes involved in the pathway are as follows: Ilv2, acetohydroxy acid synthase catalytic subunit; Ilv5, acetohydroxy acid reductoisomerase; Ilv3, dihydroxy acid dehydratase; Bat1, mitochondrial branched-chain amino acid aminotransferase; Bat2, cytosolic branched-chain amino acid aminotransferase; Leu4, $\alpha$-isopropylmalate synthase, Leu9, $\alpha$-isopropylmalate synthase; Leu1, isopropylmalate isomerase; Leu2, $\beta$-isopropylmalate dehydrogenase, KILeu4,

$\alpha$-isopropylmalate synthase, KILeu4BIS, $\alpha$-isopropylmalate synthase. PYR, pyruvate; AL, acetolactate; DHIV, dihydroxyisovalerate; KIV, ketoisovalerate; $\alpha$-IPM, $\alpha$-isopropylmalate; $\beta$-IPM, $\beta$-isopropylmalate; KIC- $\alpha$, ketoisocaproate; aCoA, Acetyl coenzyme A.

of heterodimers occurs since both ScLeu4 and ScLeu9 are located in the mitochondria. The ScLeu4-ScLeu4 isoform is inhibited by low leucine concentrations with an inhibition constant (Kii) of $0.22 \mathrm{mM}$, while the ScLeu9-ScLeu9 homodimer could be considered as leucine resistant, with a $5.44 \mathrm{mM}$ Kii, thus it is only inhibited by high intracellular concentration of this amino acid. The ScLeu4-ScLeu9 hetero-oligomeric isozyme displays a leucine inhibition constant (Kii $0.70 \mathrm{mM}$ ) intermediate to that found for the homo-dimeric isozymes (López et al., 2015). 
In a Scleu4A ScLEU9 strain, the exclusive presence of the ScLeu9-ScLeu9 leucine-resistant homodimer, results in a growth impaired phenotype, most probably due to the metabolic imbalance produced by the draining of acetyl-CoA to $\alpha$-IPM and leucine biosynthesis with the consequent depletion of other tricarboxilic acid cycle intermediates (López et al., 2015). This particular phenotype is enhanced by ScLEU9 overexpression in a Scleu4 $\Delta$ background (López et al., 2015). These results indicate that in $S$. cerevisiae, retention and further diversification of the two $\alpha$-IPMSs has resulted in a specific regulatory system that controls the leucine- $\alpha$-IPM biosynthetic pathway through the different feedback sensitivity of homomeric and heterodimeric isoforms. Here we address whether retention and further sub-functionalization of KlLEU4 and KlLEU4BIS paralogs has led to diversification of their physiological roles. We analyze the kinetic properties and sub-cellular localization of the two paralogs and their ability to complement the cognate mutations in S. cerevisiae.

\section{MATERIALS AND METHODS}

\section{Strain Transformation}

$S$. cerevisiae and K. lactis strains used in this work are described in Table 1. Yeast strains were transformed following a previously described method (Ito et al., 1983). Transformants were selected for either uracil prototrophy or leucine prototrophy on minimal medium (MM), G418 and/or nourseothricin resistance on yeast extract-peptone-dextrose (YPD)-rich medium and confirmed by PCR.

\section{Growth Conditions}

$S$. cerevisiae and $K$. lactis strains were pre-grown overnight $(16 \mathrm{~h})$ in rich media YPD. Yeast strains were routinely grown on MM containing salts, trace elements, and vitamins following the formula of yeast nitrogen base (Difco). Glucose $(2 \%, \mathrm{w} / \mathrm{v})$ or ethanol $(2 \%, \mathrm{v} / \mathrm{v})$ were used as carbon sources, and $40 \mathrm{mM}$ ammonium sulfate was used as nitrogen source. Supplements needed to satisfy auxotrophic requirements were added at $0.1 \mathrm{mg} / \mathrm{mL}$. Cells were incubated at $30^{\circ} \mathrm{C}$ with shaking (250 rpm).

\section{Construction of Kluyveromyces lactis

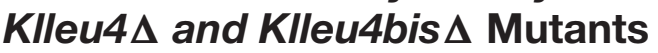

Table 1 describes the genotypes of the strains used in the present work. The single Klleu4A mutant strain (K. lactis 155-2) was constructed as follows: KlLEU4 (KLLA0F23529g) was replaced by homologous recombination in strain K. lactis 155-1 using a module containing the kanMX4 cassette flanked by 676 bp of $5^{\prime}$ untranslated region (UTR) $(-676$ to -1$)$ and 474 bp of $3^{\prime}$ UTR $(+1831$ to +2305$)$ sequences of KlLEU4. This module was amplified by overlapped extension PCR with deoxyoligonucleotides A3 and A4 (Supplementary Table S1) using a template built up by three independent modules: (i) the KlLEU4 5' UTR amplified using A3 and A5 deoxyoligonucleotides, (ii) the kanMX4 module flanked by homologous regions of the $5^{\prime}$ UTR and $3^{\prime}$ UTR of the KlLEU4 gene, which was amplified from pFA6a plasmid using deoxyoligonucleotides A6 and A7, and (iii) the KlLEU4 3' UTR amplified using deoxyoligonucleotides A8 and A4 (Supplementary Table S1). The PCR product was transformed into the $K$. lactis 155-1 strain. Transformants were selected for G418 resistance. The single Klleu4bis $\Delta$ mutant strain ( $K$. lactis 155-3) was generated as described for Klleu4 4 . Three PCR products were amplified as follows: the one corresponding to (i) the $5^{\prime}$ UTR of KlLEU4BIS (KLLA0D14201g) was amplified using deoxyoligonucleotides A9 and A10, (ii) the kanMX4 module flanked by homologous regions to the $5^{\prime}$ and $3^{\prime}$ UTR of KLLEU4BIS was amplified from pFA6a plasmid using deoxyoligonucleotides A11 and A12, and (iii) the $3^{\prime}$ UTR of KlLEU4BIS amplified using deoxyoligonucleotides A13 and A14, were used as template for an overlapped extension PCR using deoxyoligonucleotides A9 and A14. The assembled PCR product contained the

TABLE 1 | Strains used in this work.

\begin{tabular}{|c|c|c|}
\hline Strain & Relevant genotype & Source \\
\hline CLA11-700 CLA1-2 & MATa ScLEU4 ScLEU9 Scleu2:ScLEU2 Scura3 & Quezada et al., 2008 \\
\hline CLA11-701 CLA1-2-4 & MATa Scleu4 $\Delta: k a n M X 4$ ScLEU9 Scleu2:ScLEU2 Scura3 & López et al., 2015 \\
\hline CLA11-702 CLA1-2-4-9 & MATa ScLEU4 Scleu94:kanMX4 Scleu2:ScLEU2 Scura3 & This study \\
\hline CLA11-703 CLA1-2-4-9 & 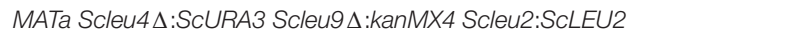 & López et al., 2015 \\
\hline CLA11-701-2 CLA12-4N & MATa Scleu4 $\Delta:$ natMX4 ScLEU9 Scleu2:ScLEU2 Scura3 & This study \\
\hline CLA11-703-2 & 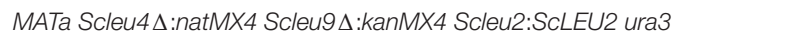 & This study \\
\hline CLA11-701-3 & Scleu4 $\Delta:$ KILEU4-yECitrine ScLEU9 Scleu2:ScLEU2 Scura3 & This study \\
\hline CLA11-701-4 & 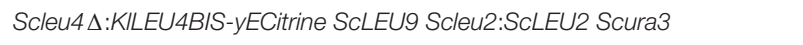 & This study \\
\hline K. lactis CBS2359ku80 & MATa ku80:/oxP ura3 & Kooistra et al., 2004 \\
\hline K. lactis $155-1$ & MAT $\alpha$ KILEU4 (KILAOF23529g) KILEU4BIS (KILAOD1421g) ade2 his3 ura3 & This study \\
\hline K. lactis $155-2$ & 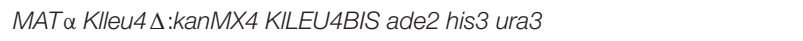 & This study \\
\hline K. lactis $155-3$ & MAT a KILEU4 Klleu4BIS $\Delta:$ kanMX4 ade2 his3 ura3 & This study \\
\hline K. lactis $155-4$ & MAT a KIleu4s:natMX4 KlleuBIS $\Delta:$ kanMX4 ade2 his3 ura3 & López et al., 2015 \\
\hline K. lactis CBS2359ku80-1 & MAT a KILEU4-yCitrine KILEU4BIS ku80:IoxP ura3 & This study \\
\hline K. lactis CBS2359ku80-2 & MAT a KILEU4 KILEU4BIS-yCitrine ku80:IoxP ura3 & This study \\
\hline
\end{tabular}


kanMX4 cassette flanked by 735 bp of $5^{\prime}$ UTR ( -735 to -1 ) and $682 \mathrm{bp}$ of $3^{\prime}$ UTR $(+1848$ to +2530$)$ of the KlLEU4BIS gene. The module was purified and used to transform the K. lactis $155-1$ strain.

\section{Construction of KILEU4-yECitrine and KILEU4BIS-yECitrine Tagged Mutants}

Deoxyoligonucleotides were designed on the basis of KlLEU4 A19 and A20 and KlLEU4BIS A21 and A22 gene sequence carrying 18 bp of $y E C i t r i n e$ (forward) or ScURA3 (reverse) (see Supplementary Table S1) and used to PCR amplify $y$ ECitrine and URA3 from plasmid pKT175 (Sheff and Thorn, 2004). The resulting modules were selectively transformed into CBS2359 $k u 80 \Delta$ (Kooistra et al., 2004), to generate strains CBS2359

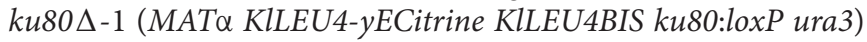

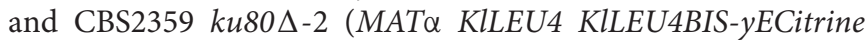
ku80:loxP ura3) (Table 1).

\section{Construction of S. cerevisiae Strains Harboring KILEU4-yECitrine and KILEU4BIS-yECitrine}

To construct $S$. cerevisiae mutant strains harboring either KlLEU4-yECitrine or KlLEU4BIS- $y$ ECitrine (Table 1), we used Scleu4A:kanMX4 ScLEU9 (CLA11-701) mutant to replace the kanMX4 cassette for either the KlLEU4-yECitrine or KlLEU4BISyECitrine generating Scleu4 $\Delta:$ KlLEU4-yECitrine ScLEU9 (CLA11-701-3) and Scleu4A:KlLEU4BIS-yECitrine ScLEU9 (CLA11-701-4) mutant strains. To obtain Scleu4A:KlLEU4yECitrine LEU9, the KlLEU4-yECitrine tagged gene (4409 bp) was PCR amplified from the genomic DNA obtained

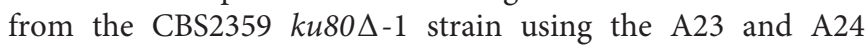
deoxyoligonucleotide pair (Supplementary Table S1). Using this amplicon as a template, a subsequent PCR was performed using A25 and A26 deoxyoligonucleotides in order to obtain a module (4529 bp) whose $5^{\prime}$ end contains an homologous region with ScLEU4 promoter from -150 to -91 and in the $3^{\prime}$ end there is an homologous region with $3^{\prime}$ UTR of ScLEU4 from +1951 to +2010 . This module was used to replace kanMX4 cassette from the strain CLA11-701 (Table 1). Transformants were selected for uracil prototrophy and confirmed by PCR using A27 and A28 deoxyoligonucleotides, the non-recombinants generated a $2330 \mathrm{bp}$ fragment, while in the transformants a $5048 \mathrm{bp}$ fragment was amplified.

Same strategy was followed to obtain Scleu4 $\triangle$ :KlLEU4BISyECitrine ScLEU9. To amplify KlLEU4BIS-yECitrine tagged gene (4468 bp) deoxyoligonucleotides A29 and A30 were used with a genomic DNA obtained from CBS2359 ku80 $\Delta-2$ strain. Subsequently, a second PCR was carried out using this fragment as a template, for this case primers A31 and A32 were used in order to generate an amplicon (4588 bp) whose $5^{\prime}$ end is homologous to ScLEU4 promoter from -150 to -91 and the $3^{\prime}$ end is homolog to the ScLEU4 3' UTR from +1951 to +2010 . This module was used to replace kanMX4 cassette from strain CLA11-701. In this case confirmation was carried out by fluorescent microscopy.

\section{Construction of YEpKD352 Plasmids Harboring KILEU4, KILEU4BIS, ScLEU4, and ScLEU9}

Standard molecular biology techniques were followed as previously described (Sambrook et al., 1989). Genes were amplified with their $5^{\prime}$ UTR sequence and cloned into plasmid YEpKD352 (pKD1 or URA3). For KlLEU4, a 2984 bp region between -933 from the start codon and +221 from the stop codon was amplified with deoxyoligonucleotides A33 and A34 (Supplementary Table S1). For KlLEU4BIS, a 2917 bp region at $-1000 \mathrm{bp}$ from the start codon and +165 from the stop codon was amplified with deoxyoligonucleotides A35 and A36. PCR modules were digested and cloned in order to generate the plasmids YEpKD352-KlLEU4 and YEpKD352-KlLEU4BIS. Genes cloned in YEpKD352 were transformed into strain $K$. lactis $155-4$ or in S. cerevisiae strains CLA1-2-4 and CLA1-2-4-9. Transformants were selected for either uracil prototrophy or leucine prototrophy on minimal medium (MM).

\section{Fluorescent Microscopy}

To confirm mitochondrial localization of KlLeu4, KlLeu4yECitrine tagged strain was stained with MitoTracker Red CMXRos (Molecular Probes) according to manufacturer's specifications. Co-localization between the MitoTracker and yECitrine was determined through sequential imaging. Confocal images were obtained using a FluoView FV1000 laser confocal system (Olympus) attached/interfaced to an Olympus IX81 inverted light microscope with a $60 \mathrm{x}$ oil-immersion objective (UPLASAPO 60x O NA:1.35), zoom x20.0 and $3.5 \mu \mathrm{m}$ of confocal aperture. The excitation and emission settings were as follows: yECitrine excitation at $488 \mathrm{~nm}$; emission $520 \mathrm{~nm}$ BF $500 \mathrm{~nm}$ range $30 \mathrm{~nm}$; MitoTracker excitation $543 \mathrm{~nm}$; emission $598 \mathrm{~nm}$, BF $555 \mathrm{~nm}$ range $100 \mathrm{~nm}$. The subsequent image processing was carried out with Olympus Fluo View FV1000 (version 1.7) software.

\section{Northern Blot Analysis}

Northern blot analysis was carried out as previously described (Struhl and Davis, 1981). Total yeast RNA was prepared from $100 \mathrm{~mL}$ aliquots of cultures grown to an optical density at $600 \mathrm{~nm}\left(\mathrm{OD}_{600 \mathrm{~nm}}\right) \sim 0.6$ on $\mathrm{MM}$ with ammonium sulfate as nitrogen source and glucose $(2 \%, w / v)$ or ethanol $(2 \%, v / v)$ as carbon source. PCR products were used as probes, for KlLEU4 a 1793 bp product was amplified with deoxyoligonucleotides A43 and A44, for KlLEU4BIS a 1644 bp product was amplified with deoxyoligonucleotides A45 and A46 (Supplementary Table S1). As internal loading standard, a probe of $477 \mathrm{bp}$ from 18S was PCR amplified with deoxyoligonucleotides A37 and A38. For ScLEU4, an 1860 bp product was amplified with deoxyoligonucleotides A39 and A40. For ScLEU9, an $1815 \mathrm{bp}$ PCR product was amplified with deoxyoligonucleotides A41 and A42. A 877 bp ScACT1 fragment amplified with deoxyoligonucleotides A47 and A48 was used as internal loading standard. Blots were scanned using the program ImageQuant 5.2 (Molecular Dynamics). 


\section{K. lactis $\alpha$-IPMSs Purification: KILeu4 and $K /$ Leu4BIS Overexpression in $E$. coli}

For KlLeu4 (KLLA0F23529) heterologous expression, the Bl21 (DE3) E. coli strain (Novagen) was transformed. KlLeu4 selected clones were grown in LB medium with $50 \mathrm{mg}$ $\mathrm{mL}^{-1}$ of kanamycin, grown at $30^{\circ} \mathrm{C}$ with shaking $(250 \mathrm{rpm})$. When cultures reached an $\mathrm{OD}_{600 \mathrm{~nm}}$ of 0.4 , expression of KlLEU4 was induced with $400 \mathrm{mmol} \mathrm{L} \mathrm{L}^{-1}$ of isopropyl- $\beta$-dthiogalactopyranoside (IPTG), incubated overnight at $16^{\circ} \mathrm{C}$ with shaking $(250 \mathrm{rpm})$, harvested by centrifugation at $1100 \mathrm{~g}$ for $15 \mathrm{~min}$, and the cellular pellet was stored at $-70^{\circ} \mathrm{C}$ until used.

For KlLeu4BIS (KLLA0D14201) heterologous expression, the Rosetta 2TM (DE3) E. coli strain (Novagen) was transformed. KlLeu4BIS selected clones were grown in LB medium with $50 \mathrm{mg}$ $\mathrm{mL}^{-1}$ of kanamycin and $70 \mathrm{mg} \mathrm{mL}-1$ of chloramphenicol, grown at $30^{\circ} \mathrm{C}$ with shaking $(250 \mathrm{rpm})$. When cultures reached an $\mathrm{OD}_{600 \mathrm{~nm}}$ of 0.6, expression of KlLeu4BIS was induced with $400 \mathrm{mmol} \mathrm{L}^{-1}$ of IPTG, incubated overnight at $16^{\circ} \mathrm{C}$ with shaking $(250 \mathrm{rpm})$, harvested by centrifugation at $1100 \mathrm{~g}$ for $15 \mathrm{~min}$, and the cellular pellet was stored at $-70^{\circ} \mathrm{C}$ until used.

\section{Preparation of Whole Cell Soluble Protein Extracts}

The cellular pellet of KlLeu4 and KlLeu4BIS strains was suspended in $20 \mathrm{~mL}$ of $50 \mathrm{mM} \mathrm{K} \mathrm{K}_{2} \mathrm{HPO}_{4}, 0.5 \mathrm{M} \mathrm{NaCl}$, $1 \mathrm{mmol} \mathrm{L}{ }^{-1}$ EDTA, $1 \mathrm{mmol} \mathrm{L}^{-1}$ dithiothreitol, $1 \mathrm{mmol} \mathrm{L}-1$ phenylmethylsulfonyl fluoride (PMSF), pH 8. Soluble extracts were obtained by sonication (Ultrasonic Processor Model: VCX130) with a tip sonicator maintaining the tubes on ice; five cycles ( $70 \%$ amplitude, 1 sec on and 1 sec off for $1 \mathrm{~min}$ ) with $1 \mathrm{~min}$ of incubation on ice between each cycle. After centrifugation at $1100 \mathrm{~g}$ for $20 \mathrm{~min}$ at $4^{\circ} \mathrm{C}$, the supernatant was stored at $4^{\circ} \mathrm{C}$.

\section{Immobilized Metal Affinity Chromatography (IMAC)}

To purify KlLeu4 protein (KLLA0F23529, syntenic), supernatant was also purified through an equilibrated nickel column (Ni-NTA Agarose, Quiagen), and following the protocol of purification of KlLeu4 with the difference that 2 extra wash steps were added. The first extra wash was 2 volumes of $30 \mathrm{mmol} \mathrm{L}^{-1}$ imidazol and the second was 2 volumes of $60 \mathrm{mmol} \mathrm{L}{ }^{-1}$ imidazol. To purify KlLeu4BIS protein (KLLA0D14201, non-syntenic), the supernatant was loaded on an equilibrated nickel column ( $\mathrm{Ni}$ NTA Agarose, Quiagen), washed with 100 volumes of lysis buffer, 50 volumes of $2 \mathrm{mmol} \mathrm{L}^{-1}$ imidazol, 2 volumes of $5 \mathrm{mmol} \mathrm{L}^{-1}$ imidazol, 2 volumes of $10 \mathrm{mmol} \mathrm{L}^{-1}$ imidazol and 2 volumes of $20 \mathrm{mmol} \mathrm{L}^{-1}$ imidazol. The protein was eluted with 2 volumes of 50,100, 200, and $300 \mathrm{mmol} \mathrm{L}^{-1}$ imidazol and stored at $4^{\circ} \mathrm{C}$ until used. KlLeu 4 and KlLeu4BIS homogeneity was verified by denaturing with a polyacrylamide gel electrophoresis (12\%, SDSPAGE) and the gel stained with Coomassie Blue. Proteins were 10-fold concentrated with AmiconR Ultra-15 10K Centrifugal Filter Devices (Millipore), and then diluted to the original sample volume with assay buffer $\left(50 \mathrm{mM} \mathrm{K}_{2} \mathrm{HPO}_{4}, \mathrm{pH}\right.$ 7.5) three "washing out" cycles were performed.

\section{$\alpha$-Isopropylmalate Synthase Enzyme Assay and Protein Determination}

Cells were grown to an $\mathrm{OD}_{600 \mathrm{~nm}} \sim 0.6$ on glucose $(2 \%$, $\mathrm{w} / \mathrm{v})$ or ethanol $(2 \%, \mathrm{v} / \mathrm{v})$ as carbon sources, and samples were collected at this point. S. cerevisiae crude extracts were obtained by disrupting the cells with glass beads in phosphate buffer A $(50 \mathrm{mM}$ phosphate buffer $\mathrm{pH}$ 7.5, $1 \mathrm{mM}$ PMSF and $1 \mathrm{mM}$ DTT). For $K$. lactis strains, lysis buffer was prepared with $50 \mathrm{mM}$ HEPES buffer $\mathrm{pH} 7.5$ and protease inhibitor cocktail tablets (Roche, cat. No. 11697 498001). The $\alpha$-isopropylmalate synthase activity was assayed with $5 \mathrm{mM}$ 5,5'-dithiobis-2-nitrobenzoic acid (DTNB, Ellman's reagent) in $1.0 \mathrm{~mL}$ of medium containing $50 \mathrm{mM}$ phosphate buffer $\mathrm{pH}$ $7.5,140 \mathrm{mM} \mathrm{KCl}, 4 \mathrm{mM} \mathrm{MgCl}, 0.25 \mathrm{mM}$ acetyl coenzyme A (acetyl-CoA) and $10 \mathrm{mM} \alpha$-ketoisovalerate $(\alpha-\mathrm{KIV})$. After $5 \mathrm{~min}$ of thermal equilibration at $30^{\circ} \mathrm{C}$ and completion of the reaction of DTNB with the contaminant CoA present in the commercial acetyl-CoA preparation, assay was started by adding cell free extract and the initial reaction rate was obtained from the absorbance change at $412 \mathrm{~nm}$ in a Varian Cary 400 spectrophotometer with a $1 \mathrm{~cm}$ path length. The 2-nitro-5thiobenzoic acid $\left(\mathrm{NTB}^{2-}\right)$ production was quantified using an extinction coefficient of $14.15 \mathrm{M}^{-1} \mathrm{~cm}^{-1}$ (Riddles et al., 1983). Specific activity is given in nmoles of CoA formed per minute. Protein concentration was determined as described previously (Lowry et al., 1951).

\section{Enzyme Kinetics and Data Analysis}

Initial velocity measurements were performed varying both substrates (for KlLeu4, 0.002-0.1 mM acetyl-CoA and 0.002$0.08 \mathrm{mM} \alpha$-KIV. While for KlLeu4BIS, $0.008-0.1 \mathrm{mM}$ acetyl-CoA and $0.01-0.25 \mathrm{mM} \alpha-\mathrm{KIV})$ and results were globally fitted to equation 1 (Eq. 1) using the Prism software. The resulting kinetic parameters are shown in Table 2.

$$
v=\frac{V_{\max }[A][B]}{K i a K b+K b[A]+K a[B]+[A][B]}
$$

Where $v$, represents initial velocity; Vmax, maximal velocity; $A$, acetyl-CoA concentration; $B, \alpha$-KIV concentration; Ka and $K b$ are the $K m$ values for $A$ and $B$ respectively and Kia is the dissociation constant for A. To study leucine inhibition, saturation curves were performed at different leucine concentrations: $0,0.01,0.02,0.06$ and 0.12 for both enzymes. At each leucine concentration, acetyl-CoA was varied (0.002$0.06 \mathrm{mM}$ ) while $\alpha$-KIV was fixed to $0.25 \mathrm{mM}$. When $\alpha$ KIV was varied (0.005-0.1 mM), acetyl-CoA concentration was fixed to $0.1 \mathrm{mM}$, the double reciprocal plot corresponded to a mixed inhibition model. Results were globally fitted using the Prism software to Eq. 2 when the varied substrate was acetyl-CoA, and to Eq. 3 when $\alpha-$ KIV was the variable substrate. In Eqs 2 and 3: I, leucine concentration; Kis is the slope associated inhibitor constant which reflects the extent of leucine dissociation to the free enzyme and Kii is the intercept associated inhibitor constant which indicates the extent of leucine dissociation to the enzyme-substrates complex. The other parameters have the same meaning as in 
TABLE 2 | K/Leu4 and K/Leu4BIS fitted kinetic parameters.

\begin{tabular}{|c|c|c|c|c|c|c|}
\hline & Vmax & Ka & Kia & $K b$ & Kis & Kii \\
\hline & $\left(\mathrm{mMol} \mathrm{min}-1 \mathrm{mg}^{-1}\right)$ & & & $(\mathrm{mM})$ & & \\
\hline KILeu4 & $3.864 \pm 0.147$ & $0.026 \pm 0.003$ & $0.017 \pm 0.005$ & $0.025 \pm 0.003$ & $0.034 \pm 0.011$ & $0.073 \pm 0.012$ \\
\hline K/Leu4BIS & $2.580 \pm 0.241$ & $0.019 \pm 0.005$ & $0.012 \pm 0.007$ & $0.055 \pm 0.013$ & $0.031 \pm 0.008$ & $0.090 \pm 0.015$ \\
\hline
\end{tabular}

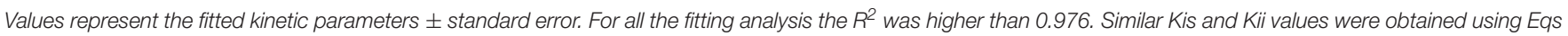
2 or 3 as described in section "Materials and Methods" section.

Eq. 1. The resulting kinetic parameters are shown in Table 2.

$$
\begin{aligned}
& v=\frac{V_{\max }[A]}{K a\left(1+\frac{K i b}{B}\right)\left(1+\frac{I}{K i s}\right)+[A]\left(1+\frac{K b}{B}\right)\left(1+\frac{I}{K i i}\right)} \\
& v=\frac{V_{\max }[B]}{K b\left(1+\frac{K i a}{A}\right)\left(1+\frac{I}{K i s}\right)+[B]\left(1+\frac{K a}{A}\right)\left(1+\frac{I}{K i i}\right)}
\end{aligned}
$$

\section{Metabolite Extraction and Analysis}

Cell extracts were prepared from exponentially growing cultures. Samples used for intracellular amino acid determination were treated as previously described (Quezada et al., 2008).

\section{Data Analysis}

Statistical analysis was performed using GraphPad Prism software version 8.0, for OS X. For each condition, the wild type KlLEU4 KlLEU4BIS strain was considered as control and one-way Analysis of Variance (ANOVA) tests was performed. Significant differences were evaluated by the Dunnett's multiple comparisons test. Results were considered significant at $p<0.05$.

\section{In silico and Bioinformatic Methods}

The following methods were used in the construction of Figure 8 and Supplementary Figures S2, S3. Peptidic sequences of putative orthologs of the S. cerevisiae ScLeu4, ScLeu9, and $K$. lactis KlLeu4 and KlLeu4BIS were obtained by BlastP from the databases of NCBI and JGI (Mycocosm). All publicly available genomes of the Saccharomycotina were mined. We included orthologs from representative members of the Taphrinomycotina and Pezizomycotina as outgroups. Alignment was carried out with MAFFT G-INS-I with default parameters ${ }^{1}$ (Kuraku et al., 2013; Katoh et al., 2019). Sequences were trimmed with trimAl, a tool for automated alignment trimming in largescale phylogenetic analysis (Capella-Gutiérrez et al., 2009). A Maximum Likelihood tree was constructed with PhyML ${ }^{2}$ (Guindon et al., 2005). Statistical test for branch support is indicated by Approximate Likelihood Ratio test (aLTR, SHlike) (Anisimova and Gascuel, 2006). The tree was visualized and edited with FigTree v1.4.3. ${ }^{3}$. Putative location (cytosolic or mitochondrial) was predicted for each sequence with Mitoprot ${ }^{4}$ (Claros and Vincens, 1996) and BacelLo 5 (Pierleoni et al., 2006).

\footnotetext{
${ }^{1}$ https://mafft.cbrc.jp/alignment/server/

${ }^{2}$ http://phylogeny.lirmm.fr/phylo_cgi/alacarte.cgi

${ }^{3}$ http://tree.bio.ed.ac.uk/software/figtree/

${ }^{4}$ https://ihg.gsf.de/ihg/mitoprot.html

${ }^{5}$ http://gpcr.biocomp.unibo.it/bacello/info.htm
}

After the tree was completed the putative localization of each protein was checked with DeepLoc- $1.0^{6}$ (Almagro Armenteros et al., 2017). The number of duplicated paralogous pairs occurring in K. lactis in relation to other species was determined using MetaPhOrs ${ }^{7}$ (Chorostecki et al., 2020).

\section{RESULTS}

\section{KILEU4/KILEU4BIS Encode $\alpha$-IsopropyImalate Synthases}

As mentioned above, the $K$. lactis genome sequence, indicates the existence of two paralogs genes presumably encoding $\alpha$ isopropylmalate synthases ( $\alpha$-IPMSs): KlLEU4 (syntenic with ScLEU4/ScLEU9) and KlLEU4BIS (non-syntenic), respectively located on chromosome XIV and XV.

To analyze the biological function of the two paralogs genes from $K$. lactis, deletion mutants in each one of the two genes (Klleu4 $\Delta$ and Klleu4bis $\Delta$ ) were constructed (section "Materials and Methods" and Table 1). Single null mutations in either one of the two genes showed wild type growth rate in cultures supplemented with ammonium as nitrogen source and glucose (Figure 2A) or ethanol (Figure 2B) as sole carbon sources. Only the double Klleu $4 \Delta$ Klleu 4 bis $\Delta$ mutant displayed leucine auxotrophy (Figures 2A,B). The addition of leucine to the media did not fully restore its growth rate to the wild type level (either on glucose or on ethanol) (Figures 2A,B), suggesting that simultaneous loss of KlLEU4 and KlLEU4BIS could result in an additional requirement(s) not fulfilled by the sole addition of leucine. This will be addressed below (see Discussion).

As expected, it was observed that when grown on glucose, leucine auxotrophy displayed by the Klleu $4 \Delta$ Klleu4bis $\Delta$ strain was complemented with plasmids harboring either one of the $K$. lactis paralogous genes (Figure 3A). However, when grown on ethanol, the double mutant transformed with plasmids harboring either KlLEU4 or KlLEU4BIS, did not fully recover the wild type phenotype, not even in the presence of leucine (Figure 3B). The $K$. lactis genes were cloned in a multicopy plasmid which could result in increased $\alpha$-IPMS activity, and thus in a metabolic imbalance due to the draining of acetyl-CoA to $\alpha$-IPM and leucine biosynthesis depleting other TCA cycle intermediates, and consequently decreasing growth rate (López et al., 2015).

\footnotetext{
${ }^{6}$ http://www.cbs.dtu.dk/services/DeepLoc/

${ }^{7}$ http://orthology.phylomedb.org/
} 

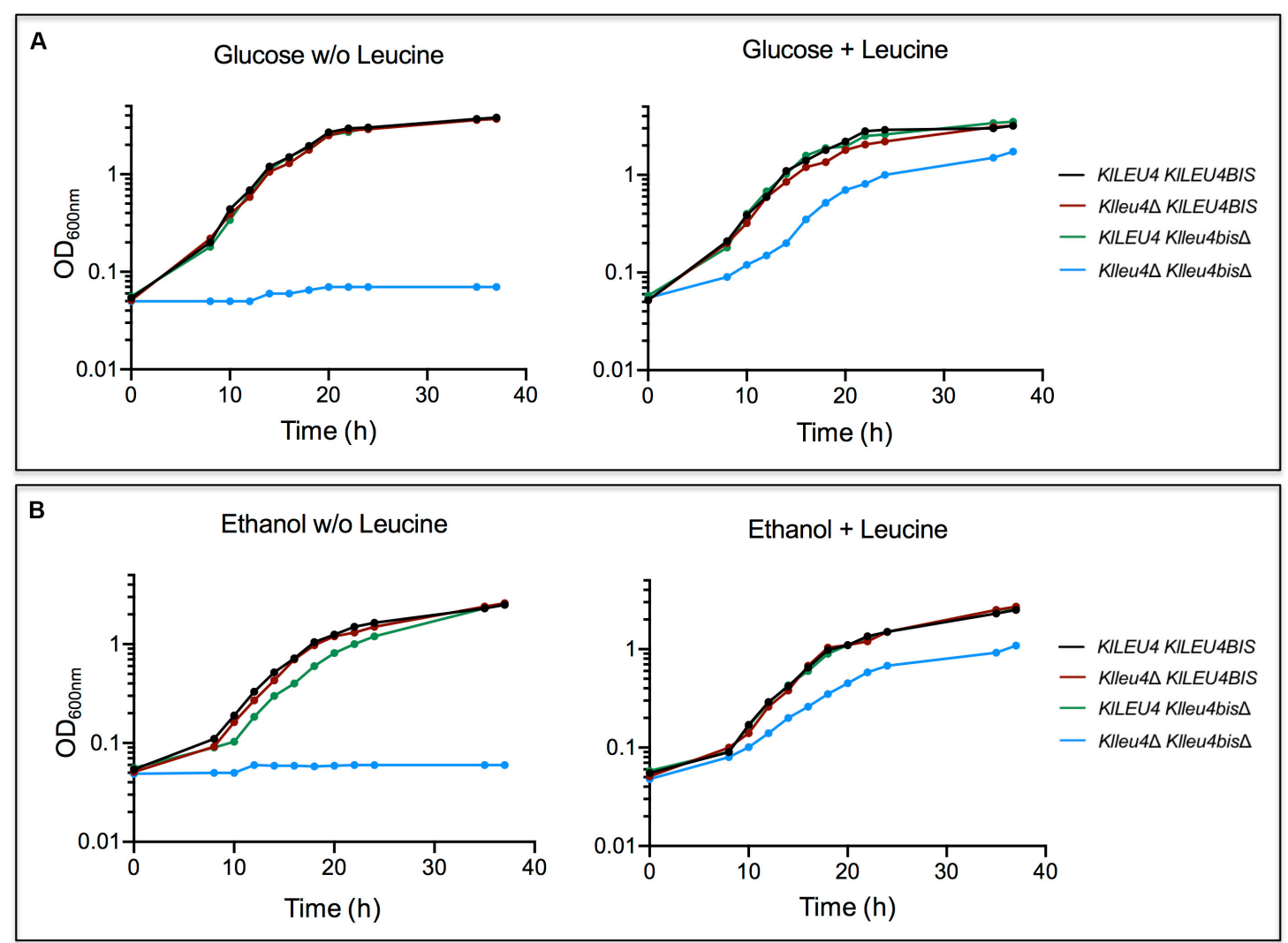

FIGURE $2 \mid K$. lactis growth curves of derived single and double KILEU4 and KILEU4B/S mutants. Strains were grown on minimal medium with $2 \%$ w/v glucose (A) or $2 \% \mathrm{v} / \mathrm{v}$ ethanol (B) as carbon sources, with or w/o leucine $(0.1 \mathrm{mg} / \mathrm{mL})$. Growth curves of wild type KILEU4 KILEU4B/S strain are shown as growth controls.

Experiments were done in duplicate.

Although, in glucose we also see this higher activity the TCA cycle is more relevant in ethanol.

As expected, in the presence of the empty vector, leucine addition allowed growth recovery, although it did not reach wild type rate, supporting the proposal that leucine does not fulfill the need of a not yet identified, additional requirement (Figure 3B).

Enzymatic activity of $\alpha$-IPMS was determined in extracts prepared from glucose or ethanol grown cultures of the wild type strain and Klleu4 $\Delta$ and Klleu4bis $\Delta$ single mutants. It was found that the Klleu4 $\Delta$ and Klleu4bis $\Delta$ single mutant strains, retained 80 or $20 \%$ of the activity found in a wild type strain, respectively. As expected, the activity fostered by the enzymes encoded in the genes cloned in the multicopy plasmids, was higher than that found in the single mutant strains (Figure 4A). In agreement with KlLeu4 and KlLeu4BIS catalytic contribution, the leucine intracellular pools were higher in the Klleu4 $\Delta$ mutant as compared to that observed in the Klleu4bis $\Delta$ mutant (Figure 4B).

Previous results showed that ScLEU4 expression is influenced by the nature of the carbon source (López et al., 2015). Thus, we analyzed whether a similar effect could be observed for KlLEU4 and KLLEU4BIS. Therefore, total RNA preparations were obtained from wild type $K$. lactis glucose or ethanol-grown cultures. Figure 4C shows that both genes have similar expression on either carbon source. We also analyzed the expression profile of each gene when its paralogous was deleted. It was found that absence of either one of the two paralogous genes did not affect expression of the remaining one (Figure 4C).

\section{K. lactis Isozymes Are Located in Different Subcellular Compartments}

The phylogeny of the $\alpha$-IPMS encoding-genes, discussed below and in Supplementary Material, strongly suggested the KlLeu4 paralog to be mitochondrial, and KlLeu4BIS to be cytosolic. Figure 5A shows this the case. Thus, it is extremely unlikely that hetero-oligomeric isoforms could form "in vivo," suggesting that the enzymatic activity detected in a wild type strain results from the addition of the independent activity of the KlLeu 4 and KlLeu4BIS homodimeric isozymes.

\section{KILeu4 and KILeu4BIS Are Leucine Sensitive}

Leucine sensitivity of the two $\alpha$-isopropylmalate synthases paralogs of $K$. lactis was analyzed. Half-maximal inhibitory concentration $\left(\mathrm{IC}_{50}\right)$ of $\mathrm{KlLeu} 4$ and $\mathrm{KlLeu} 4 \mathrm{BIS}$ was determined in whole cell extracts obtained from either Klleu4 $\Delta$ or Klleu4bis $\Delta$ single mutants. As Figure 5B shows, the two K. lactis paralogs 

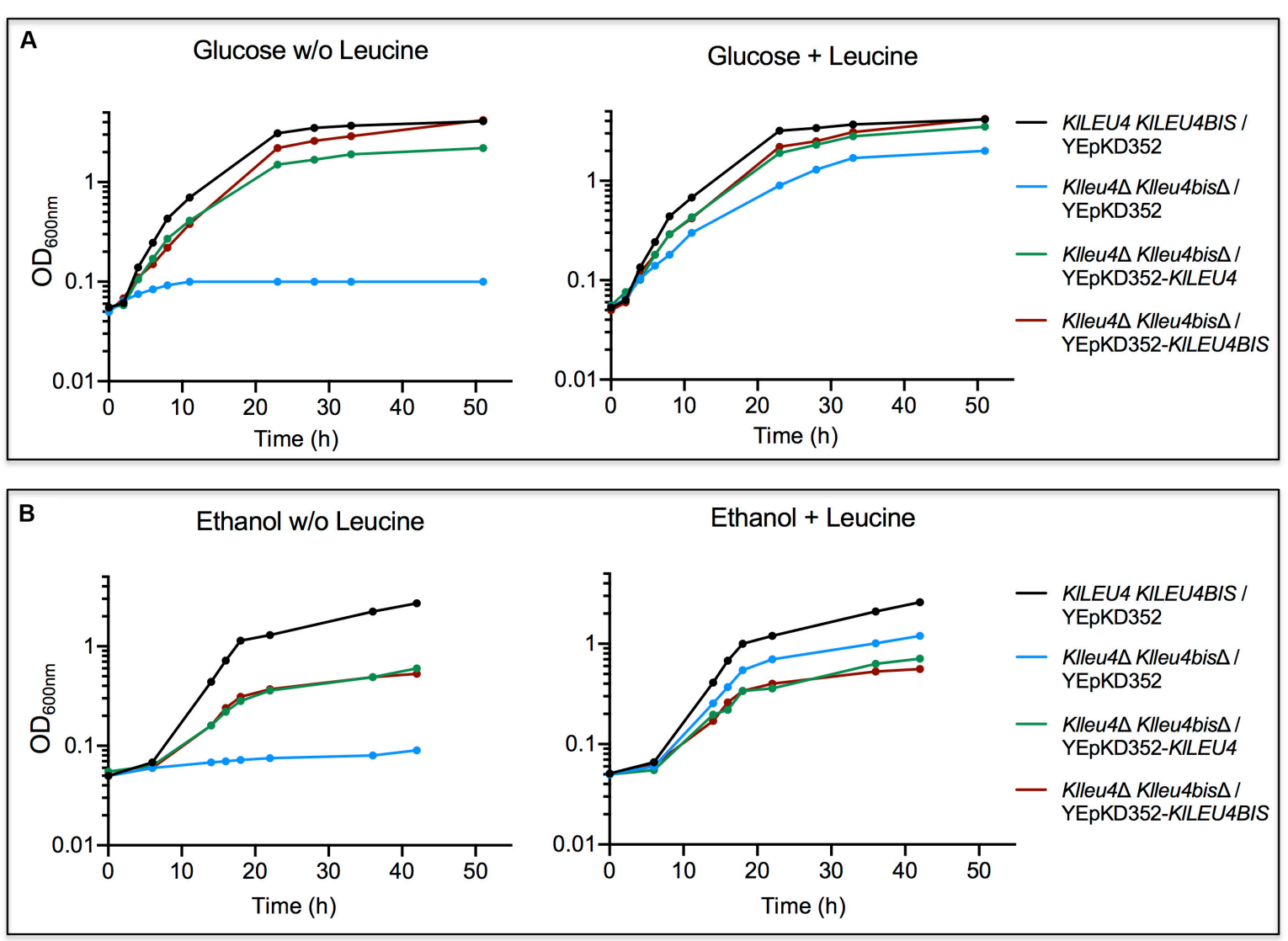

FIGURE 3|K. lactis growth curves of double Klleu4 $\Delta$ Klleu4bis $\Delta$ mutant transformed with KILEU4 or KILEU4BIS. Strains were grown on minimal medium with either $2 \% \mathrm{w} / \mathrm{v}$ glucose (A) or $2 \% \mathrm{v} / \mathrm{v}$ ethanol (B) as carbon sources, with or w/o leucine $(0.1 \mathrm{mg} / \mathrm{mL})$. Growth curves of wild type KILEU4 KILEU4B/S harboring YEpKD352 are shown as growth controls. Experiments were done in duplicate.

isozymes showed similar leucine sensitivity: KlLeu4 $0.020 \mathrm{mM}$ and KlLeu4BIS $0.037 \mathrm{mM}$.

The kinetic properties of the purified enzymes (see section "Materials and Methods") were analyzed (Supplementary Figure S1). Experimental data were fitted to Eq. 1, which corresponds to a compulsory ordered bi-bi reaction under steady state treatment (Segel, 1993). The kinetic properties of the enzymes were similar (Table 2). For both enzymes leucine behaved as a mixed inhibitor, decreasing both, the apparent affinity for the substrates and the apparent maximum enzyme reaction rate $(V \max )$. Experimental data were fitted to Eq. 2 when the acetyl-CoA concentration was varied and to Eq. 3 when the variable substrate was $\alpha$-KIV (Segel, 1993) (Table 2) (see text and equations in section "Materials and Methods"). This model predicts that leucine can bind for both isoforms either to the free enzyme or to the substrate-bound enzyme. The former process is reflected in the Kis constant whereas the latter is reflected in the Kii value (Table 2, Eqs 2 and 3 and Figure 5B). From these kinetic parameters we can thus conclude that both enzymes contribute very similarly to the control of the metabolic flux, at variance to the situation in S. cerevisiae in which the Leu9 homomeric enzyme showed a Kii value which was 24 - and 20-fold higher than the one found for the Leu4 homodimer and the Leu4-Leu9 heterodimer, respectively.

As single mutants showed wild type growth phenotypes (see above, Figure 2), KlLeu4 and KlLeu4BIS, although located in different subcellular compartments, must have a redundant role in leucine biosynthesis under the conditions tested.

\section{Heterologous Complementation of

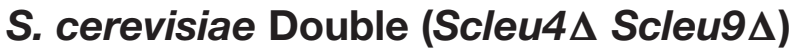 and Single (Scleu4A ScLEU9) Mutants}

Subcellular localization of the $K$. lactis orthologous isozymes heterologously expressed in $S$. cerevisiae was examined. As shown in Figure $\mathbf{6 A}$, in $S$ cerevisiae, both enzymes maintained their native localization: KlLeu4-mitochondrial and KlLeu4BIScytosolic. In an Scleu4 $\triangle$ ScLEU9 strain transformed with the KlLeu4 mitochondrial isoform, KlLeu4-ScLeu9 heterodimers could presumably be formed, thus decreasing ScLeu9-ScLeu9 homodimer concentration, hence counteracting the negative effect on growth of the ScLeu9-ScLeu9 leucine resistant isoform.

Double Scleu4A Scleu9A or single Scleu4A ScLEU9 or mutants were independently transformed with YEpKD352 plasmids each harboring KlLEU4 or KlLEU4BIS with their 

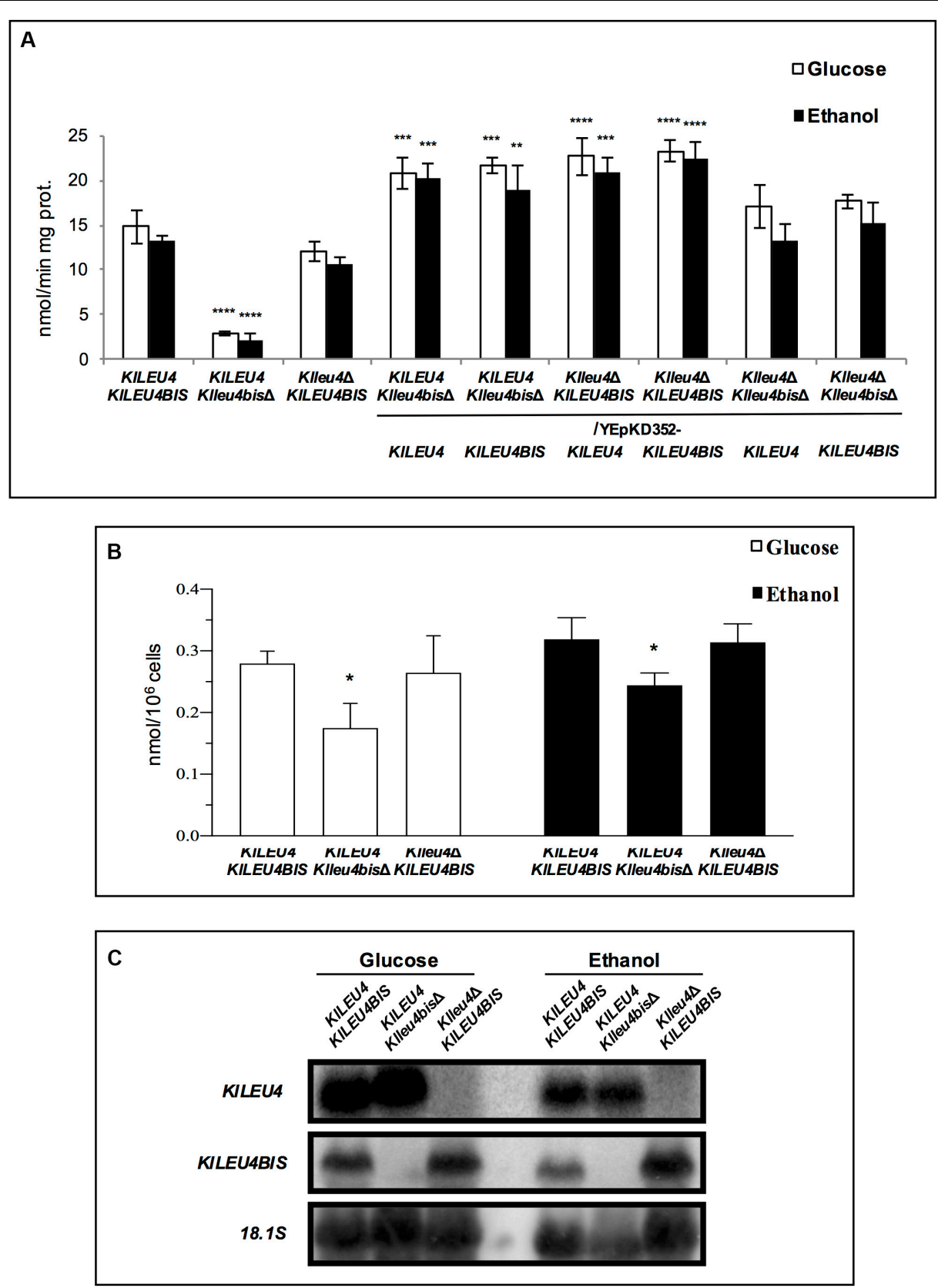

FIGURE $4 \mid \alpha$-IPMS activity, leucine pools and expression profiles determined in extracts obtained from cultures of $K$. lactis mutant strains. (A) Enzymatic activity of derived single and double Klleu4 $\Delta$ and Klleu4bis $\Delta$ mutants transformed with KILEU4 or KILEU4BIS. Wild type KILEU4 KILEU4BIS and derived single KILEU4 and KILEU4BIS mutants were used as control. Extracts were prepared from cells grown on minimal medium with either $2 \% \mathrm{w} / \mathrm{v}$ glucose or $2 \% \mathrm{v} / \mathrm{v}$ ethanol as sole carbon sources. (B) Leucine pools of wild type KILEU4 KILEU4BIS and derived single Klleu4 $\Delta$ and Klleu4bis $\Delta$ mutants were prepared as described in section "Materials and Methods." In (A,B), bars indicate mean \pm SD $(n=3),{ }^{\star} p<0.05,{ }^{* \star} p<0.01,{ }^{* \star *} p<0.001,{ }^{* \star *} p<0.0001$. (C) Northern analysis was carried out as described in section "Materials and Methods." Total Yeast RNA was prepared from $100 \mathrm{~mL}$ aliquots of cultures grown to an $\mathrm{OD}_{600 \mathrm{~nm}} \sim 0.6$ on minimal media with $2 \%$ w/v glucose or $2 \% \mathrm{v} / \mathrm{v}$ ethanol as carbon sources. Specific probes are described in section "Materials and Methods." Blots were scanned using the program ImageQuant 5.2 (Molecular Dynamics).

endogenous promoters. As controls, S. cerevisiae mutants were also transformed with ScLEU4 and ScLEU9 cloned in YEpKD352. We found that, as previously reported (López et al., 2015), when

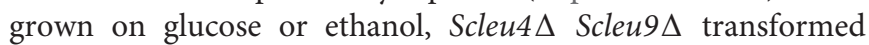
with ScLEU4 grew as well as the wild type strain. However, double mutants transformed with ScLEU9 were only partially complemented in glucose and not complemented in ethanol (Figures 7A,B). The Scleu4 $\triangle$ ScLEU9 single mutant strain, when transformed with ScLEU4 recovered a wild type phenotype, while transformants obtained with ScLEU9 showed a similar 

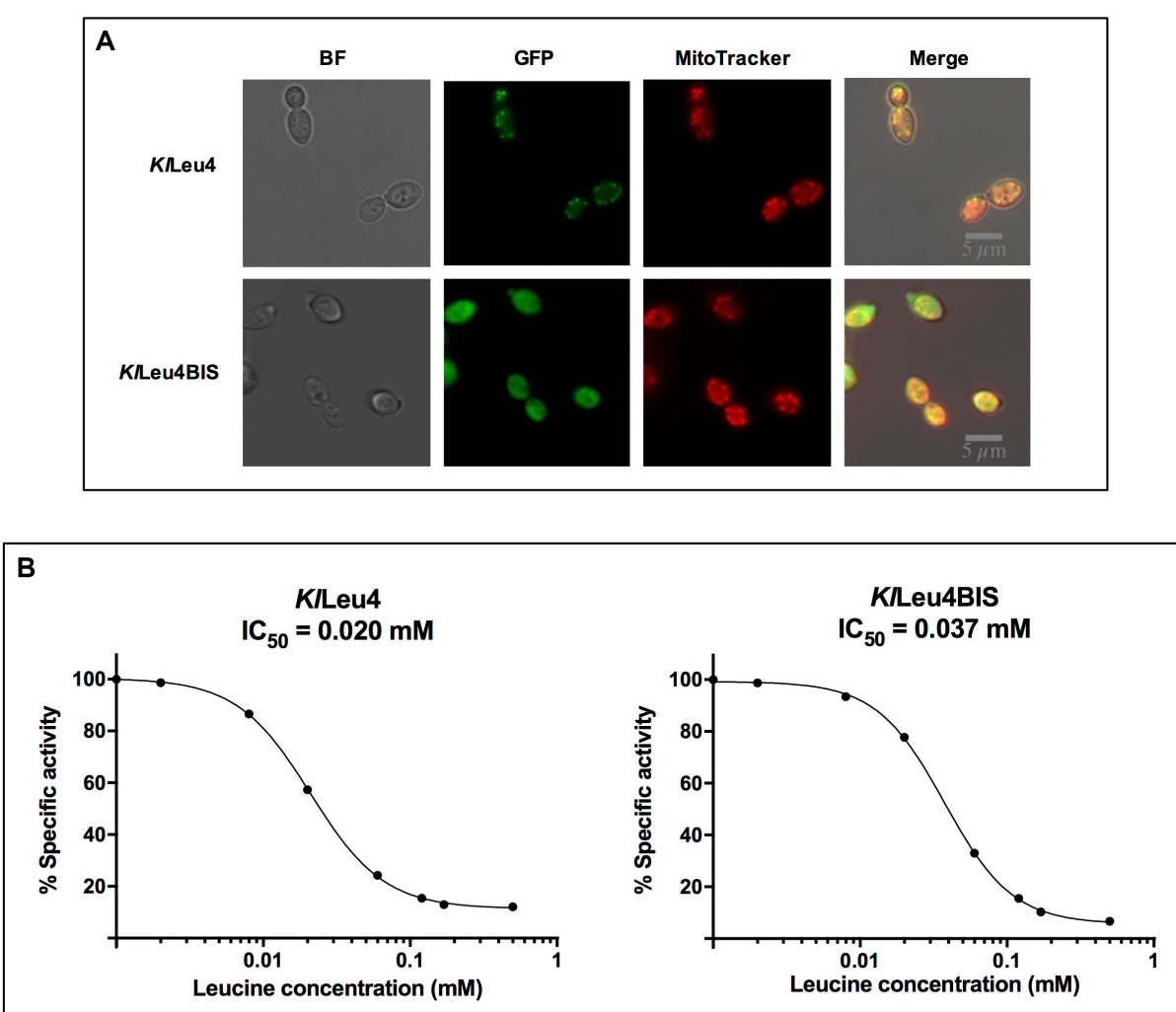

FIGURE 5 | KILeu4 and KILeu4BIS isozymes are leucine sensitive and respectively located in mitochondria and cytosol. (A) Isozyme leucine sensitivity was carried out on crude extracts as described in section "Materials and Methods." Experiments were done in duplicate. (B) Subcellular localization of KILeu4 and K/Leu4BIS paralogous proteins tagged with yECitrine using confocal microscopy. Strains were grown on minimal medium glucose (2\%, w/v) and collected during exponential phase. Representative image of multiple cells from two biological replicates.

phenotype to that displayed when transformed with the empty YEpKD352 vector (Figures 7C,D). A vector carrying KlLEU4 partially complemented the phenotype of an Scleu4A ScLEU9 strain, while one carrying KlLEU4BIS did not complement at all (Figures 7C,D).

Leucine pools were determined in the wild type strain (ScLEU4 ScLEU9), Scleu4A ScLEU9, and Scleu4A LEU9 transformed with YEpKD352 empty vector or YEpKD352-ScLEU4, ScLEU9, KlLEU4, or KlLEU4BIS. As previously reported, the Scleu4A ScLEU9 mutant accumulated a several-fold higher leucine pool, in either glucose or ethanol, as compared to that found in the wild type strain (Figure 6B). This accumulation was reverted in ScLEU4 transformants, but not in ScLEU9 transformants (López et al., 2015; Figure 6B). Surprisingly, although KlLEU4 but not KlLEU4BIS improved the growth rate of the Scleu4A ScLEU9 strain, both KlLEU4 and KlLEU4BIS transformants showed intermediate leucine accumulation, ranging between the one observed in the Scleu4 ScLEU9 mutant and that found in the Scleu4 $\triangle$ ScLEU9 transformed with ScLEU4 (Figure 6B). In an Scleu4 $\triangle$ ScLEU9 strain, LEU9 expression is several-fold enhanced (Figure 6C) this together with the leucine insensitivity of ScLeu9ScLeu9 homodimers would account for the increased leucine pool (López et al., 2015).

\section{Scleu4 $\Delta$ ScLEU9-Dependent Induced ScLEU9 Over-Expression Is Suppressed in Transformants Harboring ScLEU4, KILEU4, or KILEU4BIS}

Northern blot analysis showed that the intermediate leucine pools observed in the Scleu4 S ScLEU9, transformed with YEpKD352ScLEU4, YEpKD352-KlLEU4, or YEpKD352-KlLEU4BIS could be attributed to diminished ScLEU9 expression, as compared to that found in the Scleu4A ScLEU9/YEpKD352 (Figure 6C). Both the $K$. lactis genes mimic the ScLEU4 repressive effect on ScLEU9 expression by hitherto unidentified mechanisms. In a Scleu4 $\triangle$ ScLEU9 mutant, the reduced leucine sensitivity of ScLeu9-ScLeu9 homodimers would result in increased flux through the leucine biosynthetic pathway, depleting the pool of metabolic intermediates, leading to reduced growth. In the Scleu4 $\triangle$ ScLEU9/KlLEU4 transformed strain, ScLEU9 expression is decreased, and the reduced growth phenotype is partially alleviated. Additionally, the possible formation of a KlLeu4ScLeu9 leucine sensitive heterodimer could also contribute to the alleviated growth phenotype. Transformants obtained with KlLEU4BIS, encoding the cytosolic isoform, which is presumably unable to form heterodimers with ScLEU9, are not 

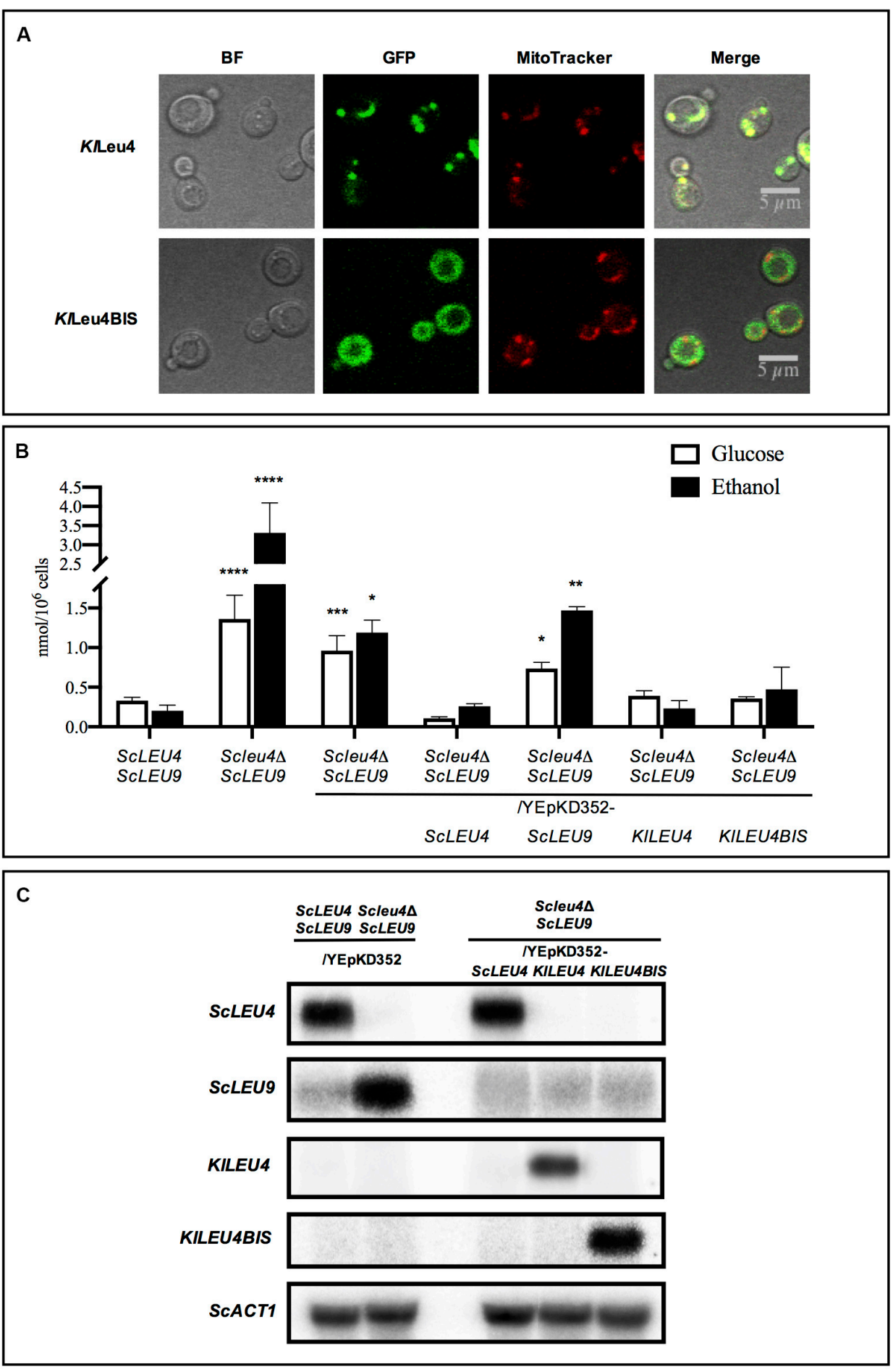

FIGURE 6 | Subcellular localization of KILeu4 and KILeu4BIS in S. cerevisiae, leucine pools and expression profiles of Scleu4 $\triangle$ ScLEU9 transformed with KILEU4 and KILEU4BIS. (A) Subcellular localization in S. cerevisiae of K/Leu4 and K/Leu4BIS paralogous proteins tagged with yECitrine using confocal microscopy. Strains were grown on minimal medium glucose $(2 \%, \mathrm{w} / \mathrm{v})$, and collected during exponential phase. Representative image of multiple cells from two biological replicates. (B) Leucine pools of wild type ScLEU4 ScLEU9 and derived single ScLEU4 mutant harboring plasmids YEpKD352-ScLEU4, YEpKD352-ScLEU9, YEpKD352-KILEU4, or YEpKD352-KILEU4BIS were prepared as described in section "Materials and Methods. Bars indicate mean $\pm \operatorname{SD}(n=3),{ }^{*} p<0.05,{ }^{* *} p<0.01$, ${ }^{* * *} p<0.001$, ${ }^{* * * *} p<0.0001$. (C) Northern analysis was carried out as described in section "Materials and Methods." Total Yeast RNA was prepared from $100 \mathrm{~mL}$ aliquots of cultures grown to an $\mathrm{OD}_{600 \mathrm{~nm}} \sim 0.6$ on minimal media with $2 \% \mathrm{w} / \mathrm{v}$ glucose as carbon source. Probes used to monitor expression for each gene are described in section "Materials and Methods." Blots were scanned using the program ImageQuant 5.2 (Molecular Dynamics). 
A

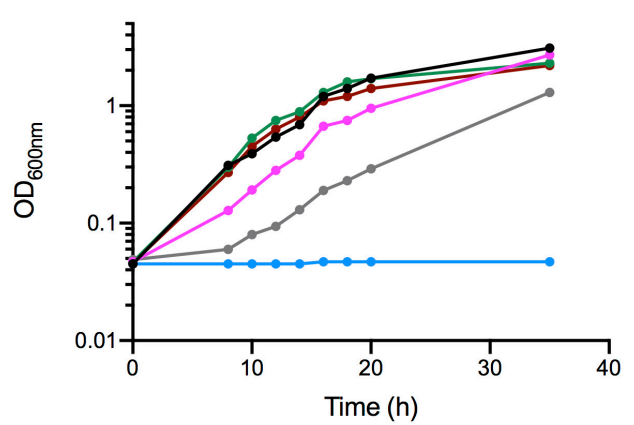

C

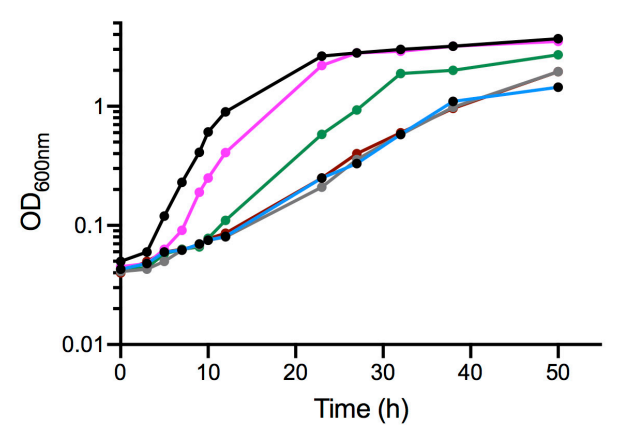

B

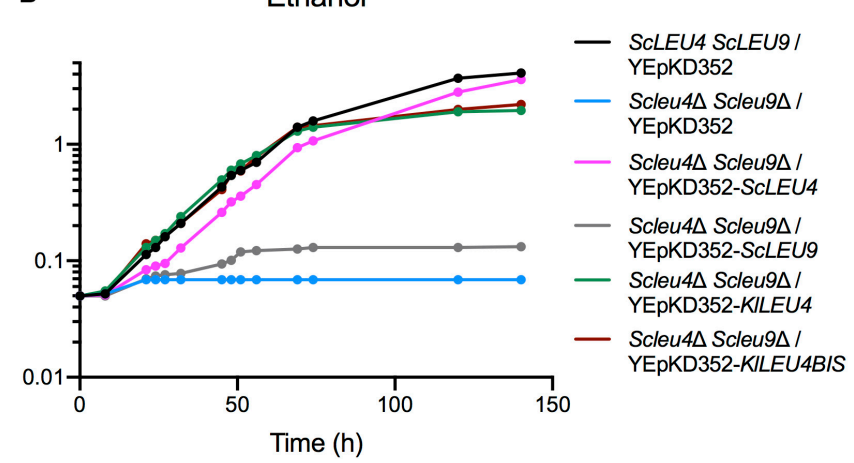

D

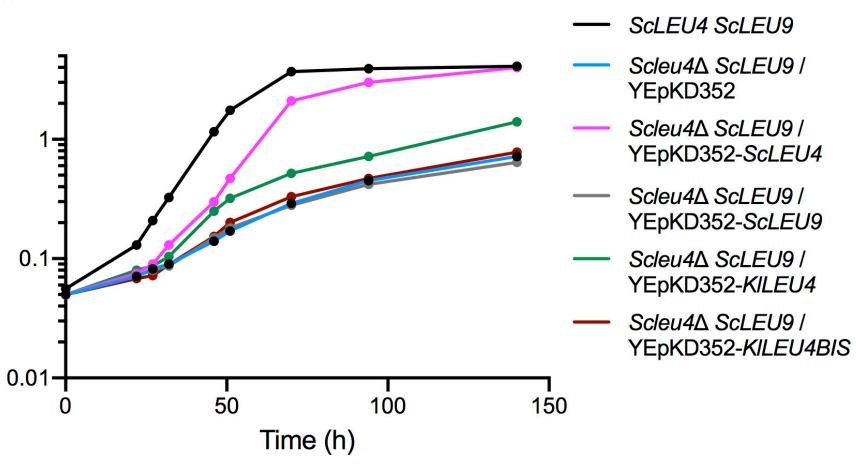

FIGURE 7 | KILEU4 and KILEU4BIS S. cerevisiae double and single mutant complementation. Growth curves of S. cerevisiae Scleu4 $\triangle$ ScLEU9 harboring plasmids YEpKD352, YEpKD352-ScLEU4, YEpKD352-ScLEU9, YEpKD352-KILEU4, or YEpKD352-KILEU4BIS. Cells were grown on minimal medium with either (A) (2\%,

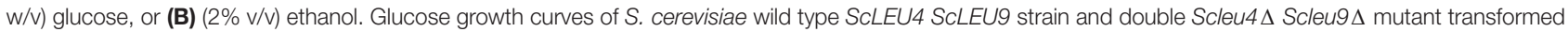
with YEpKD352-ScLEU4, YEpKD352-ScLEU9, YEpKD352-KILEU4, or YEpKD352-KILEU4BIS. Strains were grown on minimal medium with (C) (2\% w/V) glucose or (D) $(2 \% \mathrm{v} / \mathrm{v})$ ethanol. Experiments were done in duplicate.

complemented for the impaired growth phenotype although ScLEU9 expression is diminished (Figures 6C, 7C,D).

\section{Phylogeny of $\alpha$-Isopropyl Malate Synthase in the Saccharomycotina}

Isopropyl malate synthase ( $\alpha$-IPMS) is conserved throughout the tree of life (with the notable exception of metazoans) and it is present in most fungal taxa. The two mitochondrial paralogs of $S$. cerevisiae analyzed in our previous publication (López et al., 2015) originated from the WGD event, which is at the root of the sub-family of Saccharomycetaceae that includes S. cerevisiae (Wolfe and Shields, 1997; Marcet-Houben and Gabaldón, 2015; see below).

In this paper, we describe two paralogous $K$. lactis genes encoding leucine sensitive $\alpha$-IPMSs which differ in their cellular localization. The genes encoding these two isoforms must have originated by a single gene duplication preceding the WGD. We have attempted to pinpoint the origin of this duplication by constructing a phylogeny of $\alpha$-IPMSs within the Saccharomycotina. A maximum likelihood tree, including as out-groups examples of other ascomycotina (Taprhinomycotina and Pezizomycotina) is shown in Supplementary Figure S2. We have investigated the presumed location of each protein in the tree by three independent algorithms, which detect the presence/absence of a putative mitochondrial entry pre-sequence. The results are described in detail in Supplementary Data Sheet $\mathbf{1}$ and in the legend to Supplementary Figure S2 and will be briefly summarized below. Figure $\mathbf{8}$ shows schematically the crucial events of $\alpha$-IPMSs evolution in Saccharomycotina. The $\alpha$-IPMSs of Pezizomycotina (such as many plant pathogens and the model organisms Neurospora crassa and Aspergillus nidulans) and Taphrinomycotina (such as Schizosaccharomyces pombe) are presumably cytosolic (see Supplementary Data Sheet 1 for details).

Basal species of Saccharomycotina (such as Yarrowia lipolytica) include only one paralog, presumably cytosolic (PCP). A duplication, resulting in a putative mitochondrial isoform (PMP) occurred in coincidence to the emergence of the CUG-Ala clade, a monophyletic, newly classified clade of the Saccharomycotina (Shen et al., 2018, see Supplementary Data Sheet 1). The emergence of the mitochondrial isoform (PMP) occurred presumably through the inclusion of an N-terminal pre-sequence, as exemplified in Supplementary Figure S3. Most families of the Saccharomycotina include species with these two isoforms. Both S. cerevisiae and K. lactis are members of the Saccharomycetaceae. Within this family several events of loss of the presumably cytosolic paralog have occurred (Supplementary 


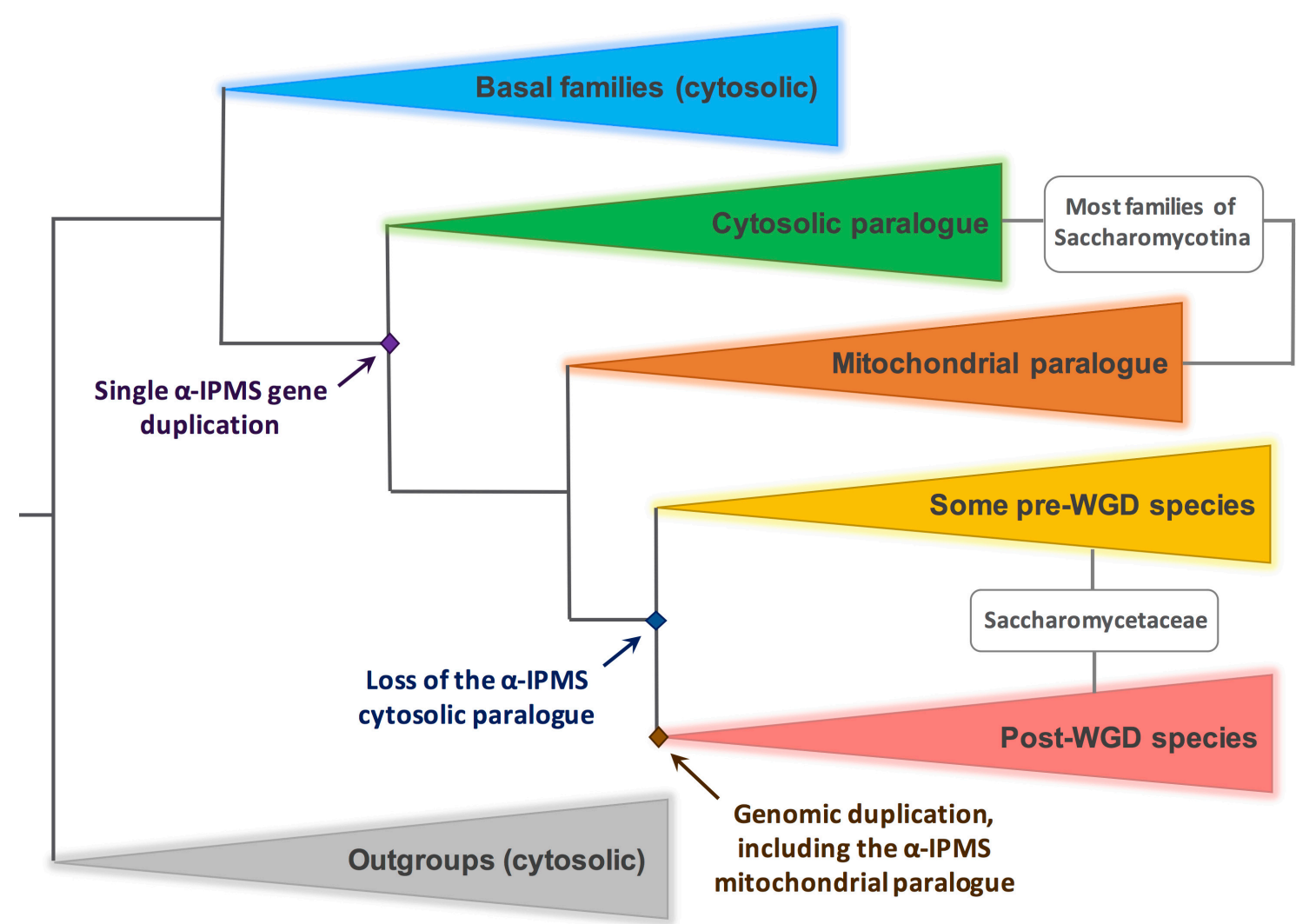

FIGURE 8 | Duplication and loss events of genes encoding $\alpha$-IPMS in the Saccharomycotina. A simplified scheme, based on the results of a comprehensive phylogeny shown in Supplementary Figure S2. The outgroup (gray triangle) include $\alpha$-IPMS genes of representative species of Taphrinomycotina and Pezizomycotina. In these clades only one paralog is extant, presumably cytosolic (see text and Supplementary Data Sheet 1). The blue triangle includes basal species of Saccharomycotina where no $\alpha$-IPMS gene duplication has occurred. Within the Saccharomycotina, a gene duplication occurred (green and brown triangles), leading to the genomes of most species comprising two isoforms, presumably cytosolic and mitochondrial respectively. KILEU4BIS and KILEU4 are included in the green and brown clade respectively. In several pre-WGD members of the Saccharomycetacea (represented by a yellow triangle), including those giving origin to the WGD, the cytosolic paralog was lost. Thus, the WGD results in two mitochondrial isoforms (red triangle). ScLEU4 and ScLEU9 originate from this duplication.

Figure S2). The WGD arose seemingly from the hybridization of two distinct species of Saccharomycetaceae, one belonging to the KLE (Kluyveromyces, Lachancea, and Eremothecium) clade, the other to the ZT (Zygosaccharomyces and Torulaspora) clade (Marcet-Houben and Gabaldón, 2015). In both clades, episodes of loss of the cytosolic paralog have occurred (Supplementary Figure S2, noticeably in Lachancea species and T. delbrueckii), thus resulting in post-WGD species including two mitochondrial isoforms but no cytoplasmic isoforms, not withstanding that further loss of one of the two mitochondrial paralogs has occurred in a few post-WGD species (such as S. pastorianus, see Supplementary Data Sheet 1 for details).

\section{DISCUSSION}

This study addresses the question of whether paralogous genes with divergent evolutionary origin constituting orthologous pairs, such as the ScLEU4/ScLEU9 vs. KlLEU4/KlLEU4BIS, follow similar or diverse functional diversification patterns.
Previous work established that putative paralogs, arising from segmental or single gene duplications are extant in species of Saccharomycetales upstream the WGD episode (Langkjaer et al., 2003; Dujon et al., 2004). Taking advantage of the MetaPhOrs database $^{7}$ (Pryszcz et al., 2011; Chorostecki et al., 2020), we have estimated that in $K$. lactis 162 gene duplications have occurred since its divergence from the basal species Yarrowia lipolytica. Incidentally, some duplicated pairs encode proteins involved in amino acid biosynthesis, such as homologs of the post WGD pairs the S. cerevisiae SER3/SER33 and LYS20/LYS21. After the $\alpha$-IPMS duplication event, which originated the K. lactis KlLEU4/KLLEU4BIS pair, episodes of loss occurred in some clades, including within the pre-WGD Saccharomyceteacea. Some species lost the cytosolic paralog. In others, through loss of the mitochondrial pre-sequence, the paralog arising from the original duplication reverted to a presumably cytosolic location. In this work we show that synthesis of $\alpha$-IPMS could occur either in the cytosol or the mitochondria. Perhaps the successive changes of localization of the $\alpha$-IPMS are related to the specific ecology and/or physiology of each species. 
ScLEU4 and ScLEU9 encoded isozymes, are both mitochondrially located; however, a fraction of ScLeu4 resides in the cytosol and could thus synthesize $\alpha$-IPM in this location. Accordingly, an oac1 $\Delta$ mutant, which is unable to transport $\alpha$-IPM from the mitochondria to the cytosol, is able to sustain a braditrophic growth feeding from the leucine synthesized in the cytosol (Marobbio et al., 2008). We have previously shown (López et al., 2015) that ScLEU4 and ScLEU9 originated from the WGD event, and have functionally diverged, resulting in three mitochondrial isoforms: the leucine-sensitive homodimeric ScLeu4-ScLeu4, the relatively leucine-resistant homodimeric ScLeu9-ScLeu9, and the heterodimeric ScLeu4-ScLeu9 displaying intermediate leucine sensitivity (López et al., 2015).

Our results indicate that K. lactis KLLEU4 and KlEU4BISencoded isozymes, which originated from a more ancient small-scale duplication event (SSD), are both leucine sensitive isozymes which, under the conditions of the present study, play a redundant role, since both genes can complement the wild type phenotype to the Klleu4 $\Delta$ Klleu4bis $\Delta$ leucine auxotrophic mutant. Divergence has been observed in their subcellular localization, KlLeu4 being mitochondrial and KlLeu4BIS cytosolic. We have asked whether each of the K. lactis orthologous pair (KlLEU4/KILEU4BIS) could complement the complex phenotype of a Scleu4 $\triangle$ ScLEU9 mutant and the leucine auxotrophy of the Scleu $4 \Delta$ Scleu9 $\Delta$ double mutant. Our results indicate that either $K$. lactis paralog can complement the leucine auxotrophy. However, the complex Scleu4 S ScLEU9 phenotype is only complemented by the KlLEU4 gene which is syntenic with ScLEU4 and ScLEU9 and encodes the mitochondrial isoform.

\section{Is the Function of the K. lactis KILEU4 and KILEU4BIS Paralogous Genes Redundant?}

The growth phenotype of the double Klleu4 $\Delta$ Klleu4bis $\Delta$ mutant is not restored to wild type levels by the sole addition of leucine, suggesting the existence of an additional requirement not supplemented by this amino acid. The transcription factor ScLeu3 activating function is positively modulated by $\alpha$-IPM (Sze et al., 1993; Boer et al., 2005). ScLeu3 ligated to $\alpha$-IPM activates transcription of various genes involved in branched chain amino acid and glutamate biosynthesis (Hu et al., 1995; Kohlhaw, 2003). Absence of $\alpha$-IPM production in the Klleu4 $\Delta$ Klleu4bis $\Delta$ double mutant could alter the presumed transcriptional role carried out by the KlLEU3 orthologous gene (KLLA0D10593g, NCBI Gene ID 2892795, ORF 52\% identity with ScLEU3). It is not far-fetched to assume that the $K$. lactis ortholog also requires $\alpha$-IPM-mediated activation. The partial, rather than complete, supplementation by leucine of the double Klleu4 $\Delta$ Klleu4bis $\Delta$

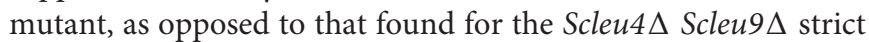
leucine auxotroph, could be due to an $\alpha$-IPM requirement, not satisfied by leucine. Although KlLEU4 and KlLEU4BIS seem to play redundant roles; it may be that in some physiological conditions the simultaneous activity of both enzymes could be necessary to attain physiological $\alpha$-IPM levels.

$K$. lactis is a strict aerobic organism, and its respiratory system is not glucose repressed (Schaffrath and Breunig, 2000), Klleu4A and Klleu4bis $\Delta$ single mutants show wild type growth rate on either glucose or ethanol as carbon and energy sources, indicating that each paralogous protein is capable to maintain energy and biomass production in aerobiosis.

The physiological function of the different localization of the first step of the pathway in S. cerevisiae and K. lactis are not obvious. KlLEU4 and KLLEU4BIS seem to play redundant roles; however, the fact that the encoded enzymes show a differential subcellular localization, which has been maintained in several families of the Saccharomycetales (see Supplementary Material) is somewhat of a paradox. Further studies will have to be carried out in order to determine whether these genes could play non-redundant roles and their relation to $\alpha$-IPM and leucine biosynthesis.

\section{The Complex Scleu4a ScLEU9 Phenotype Can Be Complemented Only by the KILeu4 Mitochondrial Isozyme}

KlLEU4 and KlLEU4BIS encoding respectively, mitochondrial and cytosolic isoforms, are able to fully complement the leucine auxotrophy displayed by Scleu4 $\Delta$ Scleu9 $\Delta$ double mutant.

We have previously shown that over-expression of ScLEU9 in a Scleu4d genetic background, results in slow growth (on either glucose or ethanol as carbon sources) and leucine accumulation. ScLeu9-ScLeu9 resistance to leucine feedback inhibition could impact flux control due to increased activity of the biosynthetic pathway (López et al., 2015). Accordingly, when the Scleu4A ScLEU9 mutant is complemented with the ScLEU4 the heterodimeric leucine-sensitive ScLeu4-ScLeu9 isozyme is formed, thus recovering the physiological feedback regulation, wild type leucine biosynthetic rate and consequently wild type growth. The KlLeu4 mitochondrial isoform is able to only partially complement the growth phenotype of the mutant Scleu4A ScLEU9, on either glucose or ethanol, partially mirroring ScLeu4 activity, while the cytosolic KlLeu4BIS isozyme did not improve Scleu4A LEU9 growth phenotype either on glucose or ethanol. Both $K$. lactis paralogs can revert the increased ScLEU9 expression to wild type levels ("Results" sections, Figure 6C). Thus, Scleu4A ScLEU9 growth rate complementation by the KlLEU4 orthologous gene could not only be due to the KlLEU4 capacity to counteract the enhanced ScLEU9 expression in a Scleu4 $\Delta$ background. Additionally, the mitochondrial localization of the KlLeu4 protein could in principle enable the formation of a heterodimeric ScLeu9KlLeu4 isoform, which may have higher leucine sensitivity than the ScLeu9-ScLeu9 homodimer, thus overcoming decreased feedback regulation. While KlLeu4BIS can prevent enhanced ScLEU9 expression, its cytosolic localization would not allow the formation of the proposed ScLeu9-KlLeu4BIS leucine sensitive heterodimers.

\section{CONCLUSION}

It can be concluded that the KlLEU4 and KLLEU4BIS paralogous pair have followed a path of functional diversification resulting in 
different subcellular localization of the encoded enzymes, which at variance from that seen in $S$. cerevisiae, do not differ in leucine sensitivity thus not affecting differentially $\alpha$-IPMS biosynthesis feedback regulation.

\section{DATA AVAILABILITY STATEMENT}

All datasets presented in this study are included in the article/Supplementary Material.

\section{AUTHOR CONTRIBUTIONS}

BA-L, JH-G, ER-O, ME-H, ML, JG, GL, and DM performed the experiments. XE-F performed and designed the experiments and contributed to the manuscript writing. MB designed the experiments and obtained the funding. CS designed the experiments, performed the phylogeny analysis, and wrote the manuscript. LR-R designed the experiments and wrote the manuscript. AG designed the experiments, wrote the manuscript, and obtained the funding. All authors contributed to the article and approved the submitted version.

\section{FUNDING}

This study was funded by the Dirección General de Asuntos del Personal Académico, UNAM, grant IN201018

\section{REFERENCES}

Almagro Armenteros, J. J., Sønderby, C. K., Sønderby, S. K., Nielsen, H., and Winther, O. (2017). DeepLoc: prediction of protein subcellular localization using deep learning. Bioinformatics 33, 3387-3395. doi: 10.1093/bioinformatics/ btx431

Anisimova, M., and Gascuel, O. (2006). Approximate likelihood-ratio test for branches: a fast, accurate, and powerful alternative. Syst. Biol. 55, 539-552. doi: 10.1080/10635150600755453

Avendaño, A., Riego, L., DeLuna, A., Aranda, C., Romero, G., Ishida, C., et al. (2005). Swi/SNF-GCN5-dependent chromatin remodeling determines induced expression of $G D H 3$, one of the paralogous genes responsible for ammonium assimilation and glutamate biosynthesis in Saccharomyces cerevisiae. Mol. Microbiol. 57, 291-305. doi: 10.1111/j.1365-2958.2005.04689.x

Beltzer, J. P., Morris, S. R., and Kohlaw, G. B. (1988). Yeast LEU4 encodes mitochondrial and nonmitochondrial forms of alpha-isopropylmalate synthase. J. Biol. Chem. 263, 368-374.

Boer, V. M., Daran, J. M., Almering, M. J., de Winde, J. H., and Pronk, J. T. (2005). Contribution of the Saccharomyces cerevisiae transcriptional regulator Leu3p to physiology and gene expression in nitrogen- and carbon limited chemostat cultures. FEMS Yeast Res. 5, 885-897. doi: 10.1016/j.femsyr.2005. 04.003

Byrne, K. P., and Wolfe, K. H. (2005). The yeast gene order browser: combining curated homology and syntenic context reveals gene fate in polyploidy species. Genome Res. 15, 1456-1461. doi: 10.1101/gr.3672305

Capella-Gutiérrez, D., Silla Martínez, J. M., and Gabaldón, T. (2009). trimAl: a tool for automated alingment trimming in large-scale. Bioinformatics 25, 1972-1973. doi: 10.1093/bioinformatics/btp348

Casalone, E., Barberio, C., Cavalieri, D., and Polsinelli, M. (2000). Identification by functional analysis of the genes encoding alpha-isopropylmalate synthase II (LEU9) in Saccharomyces cerevisiae. Yeast 16, 539-545. doi: 10.1002/(SICI) 1097-0061(200004)16:6<539::AID-YEA547<3.0.CO;2-K (http://dgapa.unam.mx), Consejo Nacional de Ciencia y Tecnología grant CB-2014-239492-B, grant from the Agencia Mexicana de Cooperación Internacional Para el Desarrollo Cooperación Científica y Tecnológica México-Italia 20152017, and grant NoCRP/MEX10-03 from the International Centre for Genetic Engineering and Biotechnology. ML had a CONACYT Master degree fellowship. XE-F was supported with a postdoctoral grant from CONACYT (CVU 420248). The funders had no role in study design, data collection and analysis, decision to publish, or preparation of the manuscript.

\section{ACKNOWLEDGMENTS}

We thank Juan Pablo Pardo for illuminating discussions during the course of this work, Toni Gabaldon for suggesting the use of MetaPhOrs, Michelle Leger for calling our attention to DeepLoc1.0. and Eduard Ocaña-Pallarés for help implementing trimAl. We also thank Javier Montalvo Arredondo and Nicolás Gómez Hernández for technical support, and Rocio Romualdo Martínez for helpful secretarial assistance.

\section{SUPPLEMENTARY MATERIAL}

The Supplementary Material for this article can be found online at: https://www.frontiersin.org/articles/10.3389/fmicb. 2020.01843/full\#supplementary-material

Cavalieri, D., Casalone, E., Bendoni, D., Fia, G., Polsinelli, M., and Barberio, C. (1999). Trifluoroleucine resistance and regulation of alpha-isopropyl malate synthase in Saccharomyces cerevisiae. Mol. Gen. Genet. 261, 152-160. doi: 10. 1007/s004380050952

Chang, L., Cunninham, T. S., Gatzeck, P. R., Chen, W., and Kohlaw, G. B. (1984). Cloning and characterization of yeast LEU4, one of two genes responsible for alpha-isopropylmalate synthesis. Genetics 108, 91-106.

Chang, L. F., Gatzek, P. R., and Kohlhaw, G. B. (1985). Total deletion of yeast LEU4: further evidence for a second alpha-isopropylmalate synthase and evidence for tight LEU4-MET4 linkage. Gene 33, 333-339. doi: 10.1016/0378-1119(85) 90241-0

Chorostecki, U., Molina, M., Pryszcz, L. P., and Gabaldón, T. (2020). MetaPhOrs 2.0: integrative, phylogeny-based inference of orthology and paralogy across the tree of life. Nucleic Acids Res. 48:gkaa282. doi: 10.1093/nar/gkaa282

Claros, M. G., and Vincens, P. (1996). Computational method to predict mitochondrially imported proteins and their targeting sequences. Eur. J. Biochem. 241, 779-786. doi: 10.1111/j.1432-1033.1996.00779.x

Colón, M., Hernández, F., López, K., Quezada, H., González, J., López, G., et al. (2011). Saccharomyces cerevisiae Batl and Bat2 aminotransferases have functionally diverged from the ancestral-like Kluyveromyces lactis orthologous enzyme. PLoS One 6:e16099. doi: 10.1371/journal.pone.0016099

Conde e Silva, N., Gonçalves, I. R., Lemaire, M., Lesuisse, E., Camadro, J. M., and Blaiseau, P. L. (2009). KlAft, the Kluyveromyces lactis ortholog of Aft 1 and Aft 2 mediates activation of the iron-responsive transcription through the PuCACCC Aft-type sequence. Genetics 183, 93-106. doi: 10.1534/genetics.109.104364

Deluna, A., Avendaño, A., Riego, L., and González, A. (2001). NADP-glutamate dehydrogenase isoenzymes of Saccharomyces cerevisiae. Purification, kinetic properties and physiological roles. J. Biol. Chem. 276, 43775-43783. doi: 10. 1074/jbc.M107986200

Dujon, B., Sherman, D., Fischer, G., Durrens, P., Casaregola, S., Lafontaine, I., et al. (2004). Genome evolution in yeasts. Nature 430, 35-44. doi: 10.1038/ nature 02579 
Force, A., Lynch, M., Pickett, F. B., Amores, A., Yan, Y. L., and Postlethwait, J. (1999). Preservation of duplicate genes by complementary, degenerative mutations. Genetics 151, 1531-1545.

González, J., López, G., Argueta, S., Escalera-Fanjul, X., El Hafidi, M., Campero-Basaldua, C., et al. (2017). Diversification of transcriptional regulation determines subfunctionalization of paralogous branched chain aminotransferases in the yeast Saccharomyces cerevisiae. Genetics 207, 975-991. doi: 10.1534/genetics.117.300290

Guindon, S., Lethiec, F., Duroux, P., and Gascuel, O. (2005). PHYML Online-a web server for fast maximum likelihood-based phylogenetic inference. Nucleic Acid Res. 33, W557-W559. doi: 10.1093/nar/gki352

Hu, Y., Cooper, T. G., and Kohlhaw, G. B. (1995). The Saccharomyces cerevisiae Leu3 protein activates expression of GDH1, a key gene in nitrogen assimilation. Mol. Cell Biol. 15, 52-57. doi: 10.1128/mcb.15.1.52

Huerta-Cepas, J., Capella-Gutiérrez, S., Pryszcz, L. P., Marcet-Houben, M., and Gabaldón, T. (2014). PhylomeDB v4: zooming into the plurality of evolutionary histories of a genome. Nucleic Acid Res. 42, D897-D902. doi: 10.1093/nar/ gkt1177

Ito, H., Fukuda, Y., Murata, K., and Kimura, A. (1983). Transformation of intact yeast cells treated with alkali cations. J. Bacteriol. 153, 163-168. doi: 10.1128/jb. 153.1.163-168.1983

Katoh, K., Rozewicki, J., and Yamada, K. D. (2019). MAFFT online service: multiple sequence alignment, interactive sequence choice and visualization. Brief. Bioinform. 20, 1160-1166. doi: 10.1093/bib/bbx108

Kellis, M., Birren, B. W., and Lander, E. S. (2004). Proof and evolutionary analysis of ancient genome duplication in the yeast Saccharomyces cerevisiae. Nature 428, 617-624. doi: 10.1038/nature02424

Kohlhaw, G. B. (2003). Leucine biosynthesis in fungi: entering metabolism through the back door. Microbiol. Mol. Biol. Rev. 67, 1-15. doi: 10.1128/mmbr.67.1.1-15. 2003

Kooistra, R., Hooykaas, P. J., and Steensma, H. Y. (2004). Efficient gene targeting in Kluyveromyces lactis. Yeast 21, 781-792. doi: 10.1002/yea.1131

Kuraku, S., Zmasek, C. M., Nishimura, O., and Katoh, K. (2013). aLeaves facilitates on-demand exploration of metazoan gene family trees on MAFFT sequence alignment server with enhanced interactivity. Nucleic Acids Res. 41, W22-W28. doi: $10.1093 /$ nar/gkt389

Langkjaer, R. B., Cliften, P. F., Johnston, M., and Piskur, J. (2003). Yeast genome duplication was followed by asynchronous differentiation of duplicated genes. Nature 421, 848-852. doi: 10.1038/nature01419

López, G., Quezada, H., Duhne, M., González, J., Lezama, M., El-Hafidi, M., et al. (2015). Diversification of paralogous $\alpha$-Isopropylmalate synthases by modulation of feedback control and hetero-oligomerization in Saccharomyces cerevisiae. Eukaryot. Cell 14, 564-577. doi: 10.1128/EC.00033-15

Lowry, O. H., Rosebrough, N. J., Farr, A. L., and Randall, R. J. (1951). Protein measurement with the Folin phenol reagent. J. Biol. Chem. 193, 265-275.

Lynch, M., Force, A., Xing, R., and Whitman, W. B. (1991). Characterization of enzymes of the branched chain amino acid biosynthetic pathway in Methanococcus spp. J. Bacteriol. 173, 2086-2092. doi: 10.1128/jb.173.6.20862092.1991

Marcet-Houben, M., and Gabaldón, T. (2015). Beyond the whole genome duplication: phylogenetic evidence for an ancient interspecies hybridization in the Baker's yeast lineage. PLoS Biol. 13:e1002220. doi: 10.1371/journal.pbio. 1002220

Marobbio, C. M., Giannuzzi, G., Paradies, E., Pierri, C. L., and Palmieri, F. (2008). alpha-Isopropylmalate, a leucine biosynthesis intermediate in yeast, is transported by the mitochondrial oxalacetate carrier. J. Biol. Chem. 283, 28445-28453. doi: 10.1074/jbc.M804637200

Marques, A. C., Vinckenbosch, N., Brawand, D., and Kaessmann, H. (2008). Functional diversification of duplicate genes through subcellular adaptation of encoded proteins. Genome Biol. 9:R54. doi: 10.1186/gb-2008-9-3-r54

Ohno, S. (1970). Evolution by Gene Duplication. Berlin: Springer-Verlag.
Pierleoni, A., Martelli, P. L., Fariselli, O., and Casadio, R. (2006). BaCelLo: a balanced subcellular localization predictor. Bioinformatics 22, e408-e416. doi: 10.1093/bioinformatics/btl222

Pryszcz, L. P., Huerta-Cepas, J., and Gabaldón, T. (2011). MetaPhOrs: orthology and paralogy predictions from multiple phylogenetic evidence using a consistency-based confidence score. Nucleic Acids Res. 39:e32. doi: 10.1093/nar/ gkq953

Quezada, H., Aranda, C., DeLuna, A., Hernández, H., Calcagno, M., MarínHernández, A., et al. (2008). Specialization of the paralogue LYS21 determines lysine biosynthesis under respiratory metabolism in Saccharomyces cerevisiae. Microbiology 154, 1656-1667. doi: 10.1099/mic.0.2008/017103-0

Riddles, P. W., Blakeley, R. L., and Zerner, B. (1983). Reassessment of Ellman's reagent. Meth. Enzymol. 91, 49-60. doi: 10.1016/s0076-6879(83) 91010-8

Roeder, P. R., and Kohlhaw, G. B. (1980). Alpha-Isopropylmalate synthase from yeast. A zinc metalloenzyme. Biochem. Biophys. Acta 613, 482-487. doi: 10. 1016/0005-2744(80)90103-5

Rojas-Ortega, E., Aguirre-López, B., Reyes-Vivas, H., González-Andrade, M., Campero-Basaldúa, J. C., Pardo, J. P., et al. (2018). Saccharomyces cerevisiae differential functionalization of presumed ScALT1 and ScALT2 alanine transaminases has been driven by diversification of pyridoxal phosphate interactions. Front. Microbiol. 9:944. doi: 10.3389/fmicb.2018.00944

Ryan, E., Tracy, J. W., and Kohlhaw, G. B. (1973). Subcellular localization of the leucine biosynthetic enzymes in yeast. J. Bacteriol. 116, 222-225. doi: 10.1128/ jb.116.1.222-225.1973

Sambrook, J., Fritsch, E. F., and Maniatis, T. (1989). Molecular Cloning: A Laboratory Manual, 2nd Edn. Cold Spring Harbor, NY: Cold Spring Harbor Laboratory.

Schaffrath, R., and Breunig, K. D. (2000). Genetics and molecular physiology of the yeast Kluyveromyces lactis. Fungal Genet. Biol. 30, 173-190. doi: 10.1006/fgbi. 2000.1221

Segel, I. H. (1993). Enzyme Kinetics: Behavior and Analysis of Rapid Equilibrium and Steady State Enzyme Systems. New York, NY: John Wiley \& Sons, 274-298.

Sheff, A. A., and Thorn, K. S. (2004). Optimized cassettes for fluorescent protein tagging in Saccharomyces cerevisiae. Yeast 21, 661-670. doi: 10.1002/yea. 1130

Shen, X. X., Opulente, D. A., Kominek, J., Zhou, X., Steenwyk, J. L., Buh, K. V., et al. (2018). Tempo and mode of genome evolution in the budding yeast subphylum. Cell 175, 1533.e20-1545.e20. doi: 10.1016/j.cell.2018.10.023

Struhl, K., and Davis, R. W. (1981). Transcription of the HIS3 gene region in Saccharomyces cerevisiae. J. Mol. Biol. 152, 535-552. doi: 10.1016/00222836(81)90267-9

Sze, J. Y., Remboutsika, E., and Kohlhaw, G. B. (1993). Transcriptional regulator Leu3 of Saccharomyces cerevisiae: separation of activator and repressor functions. Mol. Cell. Biol. 13, 5702-5709. doi: 10.1128/mcb.13.9.5702

Wolfe, K. H., and Shields, D. C. (1997). Molecular evidence for ancient duplication of the entire yeast genome. Nature 387, 708-713. doi: 10.1038/42711

Conflict of Interest: The authors declare that the research was conducted in the absence of any commercial or financial relationships that could be construed as a potential conflict of interest.

Copyright (c) 2020 Aguirre-López, Escalera-Fanjul, Hersch-González, Rojas-Ortega, El-Hafidi, Lezama, González, Bianchi, López, Márquez, Scazzocchio, Riego-Ruiz and González. This is an open-access article distributed under the terms of the Creative Commons Attribution License (CC BY). The use, distribution or reproduction in other forums is permitted, provided the original author(s) and the copyright owner(s) are credited and that the original publication in this journal is cited, in accordance with accepted academic practice. No use, distribution or reproduction is permitted which does not comply with these terms. 